\title{
Dynamical correlation functions and the related physical effects in three-dimensional Weyl/Dirac semimetals
}

\author{
Jianhui Zhou ${ }^{1}$ and Hao-Ran Chang (张浩然) ${ }^{2,3, *}$ \\ ${ }^{1}$ Department of Physics, The University of Hong Kong, Pokfulam Road, Hong Kong, China \\ ${ }^{2}$ Department of Physics, Institute of Solid State Physics and Center for Computational Sciences, \\ Sichuan Normal University, Chengdu, Sichuan 610066, China \\ ${ }^{3}$ Department of Physics, McGill University, Montreal, Quebec H3A 2T8, Canada
}

(Received 3 November 2017; revised manuscript received 30 December 2017; published 9 February 2018)

\begin{abstract}
We present a unified derivation of the dynamical correlation functions including density-density, density-current and current-current, of three-dimensional Weyl/Dirac semimetals by use of the Passarino-Veltman reduction scheme at zero temperature. The generalized Kramers-Kronig relations with arbitrary order of subtraction are established to verify these correlation functions. Our results lead to the exact chiral magnetic conductivity and directly recover the previous ones in several limits. We also investigate the magnetic susceptibilities, the orbital magnetization, and briefly discuss the impact of electron interactions on these physical quantities within the random phase approximation. Our work could provide a starting point for the investigation of the nonlocal transport and optical properties due to the higher-order spatial dispersion in three-dimensional Weyl/Dirac semimetals.
\end{abstract}

DOI: 10.1103/PhysRevB.97.075202

\section{INTRODUCTION}

Three-dimensional (3D) Weyl semimetals are one kind of new topological phase of matter and have a finite number of Weyl nodes characterized by the chirality in the Brillouin zone [1-4]. The Dirac semimetals usually host multiple pairs of Weyl nodes that are protected by both time-reversal symmetry and inversion symmetry. These Weyl nodes can be seen as monopoles, sources, and drains for Berry curvature fields in momentum space $[5,6]$. The nontrivial topology of Weyl nodes has led to a variety of exotic electromagnetic transport phenomena, such as the chiral anomaly [7-11], the static chiral magnetic effect (CME) [12-20], the dynamical CME [21-24], the topological Fermi arc states [25], and negative longitudinal magnetoresistance [26-30]. Recently, a series of experiments have made great efforts to characterize the relativistic nature of 3D Weyl/Dirac fermions and detect anomalous magnetotransport properties [31-36].

The correlation functions encode lots of essential information of the systems under the perturbations of external fields and play a critical role in investigating their physical properties [37]. The dynamical correlation functions enable us to study the responses of systems to the inhomogeneous and time-dependent external fields and the related physical effects. The density-density correlation function characterizes the dielectric behavior and is widely used to study the plasmon excitations and other many-body phenomena $[38,39]$. The current-current correlation functions are directly related to various transport properties of electrons. For example, the anomalous Hall effect is related to the off-diagonal conductivity [40], while the dynamical CME or natural optical activity is obtained from the spatially antisymmetric part of

*hrchang@mail.ustc.edu.cn off-diagonal conductivity [41]. In fact, previous works on the dynamical CME for three-dimensional (3D) Weyl fermions had mainly focused on some limits: the leading-order part of the chiral magnetic conductivity [21] and the next-toleading-order hard dense loop approximation [22]. However, the general frequency- and momentum-dependent correlation functions for 3D Weyl/Dirac semimetals are still lacking and deserve detailed derivations. The prime aim of this paper is to derive the dynamical correlation functions of 3D Weyl/Dirac semimetals in a unified framework of the Passarino-Veltman reduction scheme (PVRS) [42], which is capable of reducing the tensor integral over loop energy-momentum to basic scalar integrals based on the tensor structure imposed by Lorentz covariance.

In this paper, the dynamical correlation functions of Weyl/Dirac semimetals are derived by use of the PVRS at zero temperature. We establish the generalized Kramers-Kronig relations with $n$ th-order subtraction to verify these correlation functions. The gauge invariance of these correlation functions is also clarified. We obtain the exact analytical chiral magnetic conductivity and make a comparison with the previous results in several limits. In addition, we explore the magnetic susceptibilities, the orbital magnetization, and briefly discuss the impact of electron interactions on these quantities within the random phase approximation (RPA).

This paper is organized as follows. In Sec. II, we outline the effective Hamiltonian and introduce the correlation functions. In Sec. III, we calculate the correlation functions by using the PVRS. In Sec. IV, we establish the generalized KramersKronig relation and apply them to the correlation functions. In Sec. V, the optical conductivity is recovered. In Sec. VI, the exact chiral magnetic conductivity is derived and some limits are discussed. In Sec. VII, we evaluate the magnetic susceptibilities, the orbital magnetization, and renormalizations of these physical quantities due to electron interactions. 
In Sec. VIII, the main results of this paper are summarized. Finally, we give the detailed calculations in the Appendices.

\section{THE HAMILTONIAN AND CORRELATION FUNCTIONS}

We start from the effective Hamiltonian for a pair of isotropic Weyl nodes with opposite chirality [43]

$$
\mathcal{H}_{\chi}=\chi v_{F} \hbar \sigma^{\alpha}\left(k^{\alpha}+\chi b^{\alpha}\right)-\sigma^{0} \mu_{\chi},
$$

where $\sigma^{\alpha}$ with $\alpha=x, y, z$ are the Pauli matrices and $\sigma^{0}$ is the unit matrix. The velocity operator is given as $v^{\alpha}=$ $\partial \mathcal{H}_{\chi} / \partial\left(\hbar k^{\alpha}\right)=\chi v_{F} \sigma^{\alpha}$, with $v_{F}$ being the effective velocity. $\mu_{\chi}=\mu_{0}+\chi b_{0}$ is the chirality-dependent chemical potential, and $\chi= \pm$ denotes the chirality of the Weyl node. $\mu_{0}$ is the chemical potential at equilibrium. $b_{0}$ refers to the chiral chemical potential induced by the chiral anomaly or the energy difference between the two Weyl nodes. $\boldsymbol{b}$ measures the spacing of Weyl nodes with opposite chirality from the time-reversal symmetry breaking. In particular, for $b_{0}=0$ and $\boldsymbol{b}=0$, the effective Hamiltonian in Eq. (1) reduces to a minimal model for the Dirac semimetals. Hereafter, we shall set $\hbar=v_{F}=1$ unless specified otherwise.

The corresponding Matsubara Green's function of Weyl fermions near the Weyl node $\chi, G_{\chi}\left(\boldsymbol{k}, i \Omega_{n}\right)=\left(i \Omega_{n} \sigma^{0}-\mathcal{H}_{\chi}\right)^{-1}$, is given as

$$
G_{\chi}\left(\boldsymbol{k}, i \Omega_{n}\right)=\frac{\left(i \Omega_{n}+\mu_{\chi}\right) \sigma^{0}+\chi \sigma^{\alpha}\left(k^{\alpha}+\chi b^{\alpha}\right)}{\left(i \Omega_{n}+\mu_{\chi}\right)^{2}-(\boldsymbol{k}+\chi \boldsymbol{b})^{2}},
$$

where $\Omega_{n}=(2 n+1) \pi / \beta_{T}$ is the fermionic Matsubara frequency with $\beta_{T}=1 / k_{B} T$. In this paper, we mainly focus on the following case [44]:

$$
G_{\chi}\left(\boldsymbol{k}, i \Omega_{n}\right)=\frac{\left(i \Omega_{n}+\mu_{\chi}\right) \sigma^{0}+\chi \sigma^{\alpha} k^{\alpha}}{\left(i \Omega_{n}+\mu_{\chi}\right)^{2}-k^{2}},
$$

with $k^{2}=\left(k^{x}\right)^{2}+\left(k^{y}\right)^{2}+\left(k^{z}\right)^{2}$.

The total correlation function for 3D Weyl/Dirac semimetals is a summation of these chirality-dependent correlation functions

$$
\Pi^{\mu v}\left(\boldsymbol{q}, i \omega_{m}\right)=\sum_{\chi= \pm} \Pi^{\mu v}\left(\boldsymbol{q}, i \omega_{m}, \chi\right)
$$

where the indices $\mu, \nu=0$ are for the time component, while $\mu, \nu=x, y, z$ represent the spatial components. In general, there are three kinds of chirality-dependent correlation functions. The first kind is the chirality-dependent density-density correlation function

$$
\begin{aligned}
\Pi^{00}\left(\boldsymbol{q}, i \omega_{m}, \chi\right)= & \frac{1}{\mathcal{V}} \sum_{\boldsymbol{k}} \frac{1}{\beta_{T}} \sum_{i \Omega_{n}} \operatorname{Tr}\left[\sigma^{0} G_{\chi}\left(\boldsymbol{k}, i \Omega_{n}\right)\right. \\
& \left.\times \sigma^{0} G_{\chi}\left(\boldsymbol{k}+\boldsymbol{q}, i \Omega_{n}+i \omega_{m}\right)\right],
\end{aligned}
$$

where $\mathrm{Tr}$ acts over the internal degrees of freedom (pseudospin or spin). It had been derived in detail in Refs. [45-47] and used to extensively investigate the plasmon excitations [47-54] and dynamics of phonons [55-57] in 3D Weyl/Dirac semimetals. The second kind is the chirality-dependent current-current correlation function

$$
\begin{aligned}
\Pi^{\alpha \beta}\left(\boldsymbol{q}, i \omega_{m}, \chi\right)= & \frac{1}{\mathcal{V}} \sum_{\boldsymbol{k}} \frac{1}{\beta_{T}} \sum_{i \Omega_{n}} \operatorname{Tr}\left[\left(\chi \sigma^{\alpha}\right) G_{\chi}\left(\boldsymbol{k}, i \Omega_{n}\right)\right. \\
& \left.\times\left(\chi \sigma^{\beta}\right) G_{\chi}\left(\boldsymbol{k}+\boldsymbol{q}, i \Omega_{n}+i \omega_{m}\right)\right]
\end{aligned}
$$

Note that the spatially antisymmetric part of the off-diagonal correlation functions $\Pi_{A}^{\alpha \beta} \equiv \frac{1}{2}\left(\Pi^{\alpha \beta}-\Pi^{\beta \alpha}\right)$ has been used to study the dynamical CME in several limits [21,22]. If $\sigma^{\alpha}$ stands for the real spin degree of freedom of electrons, the current-current correlation functions are proportional to the dynamical spin susceptibilities, which govern the RudermanKittel-Kasuya-Yosida interaction of magnetic impurities and the spin textures $[43,58]$.

Finally, the chirality-dependent density-current correlation functions are given by

$$
\begin{aligned}
\Pi^{0 \alpha}\left(\boldsymbol{q}, i \omega_{m}, \chi\right)= & \frac{1}{\mathcal{V}} \sum_{\boldsymbol{k}} \frac{1}{\beta_{T}} \sum_{i \Omega_{n}} \operatorname{Tr}\left[\sigma^{0} G_{\chi}\left(\boldsymbol{k}, i \Omega_{n}\right)\right. \\
& \left.\times\left(\chi \sigma^{\alpha}\right) G_{\chi}\left(\boldsymbol{k}+\boldsymbol{q}, i \Omega_{n}+i \omega_{m}\right)\right] \\
\Pi^{\alpha 0}\left(\boldsymbol{q}, i \omega_{m}, \chi\right)= & \frac{1}{\mathcal{V}} \sum_{\boldsymbol{k}} \frac{1}{\beta_{T}} \sum_{i \Omega_{n}} \operatorname{Tr}\left[\left(\chi \sigma^{\alpha}\right) G_{\chi}\left(\boldsymbol{k}, i \Omega_{n}\right)\right. \\
& \left.\times \sigma^{0} G_{\chi}\left(\boldsymbol{k}+\boldsymbol{q}, i \Omega_{n}+i \omega_{m}\right)\right]
\end{aligned}
$$

which is critical to the gauge invariance among these correlation functions. In the following, we evaluate all of the chirality-dependent correlation functions $\Pi^{\mu \nu}\left(\boldsymbol{q}, i \omega_{m}, \chi\right)$ by utilizing the PVRS [42].

\section{CORRELATION FUNCTIONS FROM THE PASSARINO-VELTMAN REDUCTION SCHEME}

The essential spirit of the PVRS is to reduce the tensor integral over loop energy-momentum to a set of basic scalar integrals by considering the tensor structure imposed by Lorentz covariance [42]. This scheme is widely used in the calculations of perturbative corrections in high-energy physics. For 3D Weyl/Dirac semimetals, the Lorentz covariance is evident such that the PVRS is applicable to calculations of their correlation functions.

Utilizing the PVRS, summing over Matsubara frequency $\Omega_{n}$, and performing the analytical continuum $i \omega_{m} \rightarrow \omega+i \eta$ with $\eta$ being a positive infinitesimal, one finally rewrites these chirality-dependent correlation functions in terms of a set of scalar functions (see Appendix A). First, the chiralitydependent density-density correlation function

$$
\Pi^{00}(\boldsymbol{q}, \omega, \chi)=\frac{1}{2}\left[A_{0}+B_{a}+q^{2} B_{1}\right] .
$$

Second, the chirality-dependent current-current correlation function $\Pi^{\alpha \beta}(\boldsymbol{q}, \omega, \chi)$ is given as

$$
\begin{aligned}
\Pi^{\alpha \beta}(\boldsymbol{q}, \omega, \chi)= & F_{T}(\boldsymbol{q}, \omega, \chi)\left(\delta^{\alpha \beta}-\frac{q^{\alpha} q^{\beta}}{q^{2}}\right)+F_{L}(\boldsymbol{q}, \omega, \chi) \\
& \times \frac{q^{\alpha} q^{\beta}}{q^{2}}+i \chi F_{A}(\boldsymbol{q}, \omega, \chi) \varepsilon^{\alpha \beta \gamma} q^{\gamma}
\end{aligned}
$$

with

$$
\begin{gathered}
F_{T}(\boldsymbol{q}, \omega, \chi)=\frac{1}{2}\left[A_{0}-B_{b}-q^{2} B_{1}\right], \\
F_{L}(\boldsymbol{q}, \omega, \chi)=\frac{1}{2}\left[A_{0}-B_{a}+2 B_{b}+q^{2} B_{1}\right], \\
F_{A}(\boldsymbol{q}, \omega, \chi)=-\frac{1}{2}\left[C_{0}+C_{1}-D_{1}\right],
\end{gathered}
$$


where $\varepsilon^{\alpha \beta \gamma}$ is the Levi-Civita symbol and $\varepsilon^{x y z}=1$. $F_{T / L}(\boldsymbol{q}, \omega, \chi)$ refers to the transverse/longitudinal component of the symmetric part of the current-current correlation functions. Third, the chirality-dependent density-current correlation functions $\Pi^{0 \alpha / \alpha 0}(\boldsymbol{q}, \omega, \chi)$ are given as

$$
\Pi^{0 \alpha}(\boldsymbol{q}, \omega, \chi)=\Pi^{\alpha 0}(\boldsymbol{q}, \omega, \chi)=F_{I}(\boldsymbol{q}, \omega, \chi) q^{\alpha},
$$

with

$$
F_{I}(\boldsymbol{q}, \omega, \chi)=\frac{1}{2}\left[C_{0}+C_{1}+D_{1}\right] .
$$

Since $\Pi^{0 \alpha}(\boldsymbol{q}, \omega, \chi)$ and $\Pi^{\alpha 0}(\boldsymbol{q}, \omega, \chi)$ are parallel to $q^{\alpha}$, neither of them contributes to the orbital magnetization.

For convenience, we shall decompose the correlation function $\Pi^{\mu \nu}(q, \omega, \chi)$ into two parts, $\Pi^{\mu \nu}(\boldsymbol{q}, \omega, \chi)=$ $\Pi_{-}^{\mu v}(\boldsymbol{q}, \omega, \chi)+\Theta\left(\left|\mu_{\chi}\right|\right) \Pi_{+}^{\mu v}(\boldsymbol{q}, \omega, \chi)$, where $\Pi_{-/+}^{\mu \nu}(\boldsymbol{q}, \omega, \chi) \mathrm{de}-$ notes the intrinsic/extrinsic correlation functions. After lengthy and complicated calculations, one finds the explicit expressions of the seven scalar integrals $A_{0}^{ \pm}, B_{1}^{ \pm}, B_{a}^{ \pm}, B_{b}^{ \pm}, C_{0}^{ \pm}, C_{1}^{ \pm}$, and $D_{1}^{ \pm}$at zero temperature (see Appendices B and C) [59]. Accordingly, one can obtain the intrinsic parts of the chiralitydependent correlation functions with $\mu_{\chi}=0$,

$$
\begin{gathered}
\operatorname{Im} \Pi_{-}^{00}(\boldsymbol{q}, \omega, \chi)=-\frac{q^{2}}{24 \pi} \Theta(\omega-q), \\
\operatorname{Re} \Pi_{-}^{00}(\boldsymbol{q}, \omega, \chi)=-\frac{q^{2}}{24 \pi^{2}} \log \left|\frac{4 \Lambda^{2}}{q^{2}-\omega^{2}}\right|,
\end{gathered}
$$

$$
\begin{gathered}
\operatorname{Im} F_{T}^{-}(\boldsymbol{q}, \omega, \chi)=\frac{q^{2}-\omega^{2}}{24 \pi} \Theta(\omega-q), \\
\operatorname{Re} F_{T}^{-}(\boldsymbol{q}, \omega, \chi)=-\frac{1}{24 \pi^{2}}\left(4 \Lambda^{2}-\frac{3}{5} q^{2}\right) \\
+\frac{q^{2}-\omega^{2}}{24 \pi^{2}} \log \left|\frac{4 \Lambda^{2}}{q^{2}-\omega^{2}}\right|, \\
\operatorname{Im} F_{L}^{-}(\boldsymbol{q}, \omega, \chi)=\frac{-\omega^{2}}{24 \pi} \Theta(\omega-q), \\
\operatorname{Re} F_{L}^{-}(\boldsymbol{q}, \omega, \chi)=-\frac{1}{24 \pi^{2}}\left(4 \Lambda^{2}-\frac{4}{5} q^{2}\right) \\
\operatorname{Im} F_{A}^{-}(\boldsymbol{q}, \omega, \chi)=0, \\
\operatorname{Re} F_{I}^{-}(\boldsymbol{q}, \omega, \chi)=-\frac{\omega^{2}}{24 \pi^{2}} \log \left|\frac{4 \Lambda^{2}}{q^{2}-\omega^{2}}\right|, \\
\operatorname{Re} F_{A}^{-}(\boldsymbol{q}, \omega, \chi)=\frac{\omega}{24 \pi^{2}}, \\
\operatorname{Im} F_{I}^{-}(\boldsymbol{q}, \omega, \chi)=-\frac{\omega}{24 \pi} \Theta(\omega-q), \\
q^{2}-\omega^{2} \mid
\end{gathered}
$$

and the extrinsic parts for $\mu_{\chi}>0$ (those for $\mu_{\chi}<0$ can be obtained from Appendix F),

$$
\left.\left.\begin{array}{rl}
\operatorname{Im} \Pi_{+}^{00}(\boldsymbol{q}, \omega, \chi)= & -\frac{1}{64 \pi}\left\{\Theta(q-\omega) \sum_{\lambda= \pm} \lambda \Theta\left(\mu_{\chi}-\frac{q-\lambda \omega}{2}\right) G_{S}(q, \lambda \omega)-\Theta(\omega-q)\right. \\
& \left.\times\left[\frac{8}{3} q^{2} \Theta\left(\mu_{\chi}-\frac{\omega+q}{2}\right)+\tilde{\Theta}\left(\frac{\omega+q}{2}-\mu_{\chi}\right) \Theta\left(\mu_{\chi}-\frac{\omega-q}{2}\right) G_{S}(-q,-\omega)\right]\right\} \\
& \operatorname{Re} \Pi_{+}^{00}(\boldsymbol{q}, \omega, \chi)=-\frac{\mu_{\chi}^{2}}{3 \pi^{2}}+\frac{1}{64 \pi^{2}} \sum_{\lambda, \lambda^{\prime}= \pm} G_{S}\left(\lambda q, \lambda^{\prime} \omega\right) H\left(\lambda q, \lambda^{\prime} \omega\right) \\
\operatorname{Im} F_{T}^{+}(\boldsymbol{q}, \omega, \chi)=- & \frac{1}{64 \pi}\left\{\Theta(q-\omega) \sum_{\lambda= \pm} \lambda \Theta\left(\mu_{\chi}-\frac{q-\lambda \omega}{2}\right) G_{M}(q, \lambda \omega)-\Theta(\omega-q)\right. \\
\times & \left.\left.-\frac{8}{3}\left(q^{2}-\omega^{2}\right) \Theta\left(\mu_{\chi}-\frac{\omega+q}{2}\right)+\tilde{\Theta}\left(\frac{\omega+q}{2}-\mu_{\chi}\right) \Theta\left(\mu_{\chi}-\frac{\omega-q}{2}\right) G_{M}(-q,-\omega)\right]\right\} \\
& \operatorname{Re} F_{T}^{+}(\boldsymbol{q}, \omega, \chi)=\frac{\mu_{\chi}^{2}\left(q^{2}+2 \omega^{2}\right)}{12 \pi^{2} q^{2}}+\frac{1}{64 \pi^{2}} \sum_{\lambda, \lambda^{\prime}= \pm} G_{M}\left(\lambda q, \lambda^{\prime} \omega\right) H\left(\lambda q, \lambda^{\prime} \omega\right) \\
\operatorname{Im} F_{L}^{+}(\boldsymbol{q}, \omega, \chi)= & -\frac{1}{64 \pi}\left\{\Theta(q-\omega) \sum_{\lambda= \pm} \lambda \Theta\left(\mu, \frac{q-\lambda \omega}{2}\right) G_{N}(q, \lambda \omega)-\Theta(\omega-q)\right. \\
& \quad \operatorname{Re} F_{L}^{+}(\boldsymbol{q}, \omega, \chi)=-\frac{\mu_{\chi}^{2} \omega^{2}}{3 \pi^{2} q^{2}}+\frac{1}{64 \pi^{2}} \sum_{\lambda, \lambda^{\prime}= \pm} G_{N}\left(\lambda q, \lambda^{\prime} \omega\right) H\left(\lambda q, \lambda^{\prime} \omega\right), \\
& \left.\times\left[\frac{8}{3} \omega^{2} \Theta\left(\mu_{\chi}-\frac{\omega+q}{2}\right)+\tilde{\Theta}\left(\frac{\omega+q}{2}-\mu_{\chi}\right) \Theta\left(\mu_{\chi}-\frac{\omega-q}{2}\right) G_{N}(-q,-\omega)\right]\right\}
\end{array}\right]\right\}
$$




$$
\begin{aligned}
\operatorname{Im} F_{A}^{+}(\boldsymbol{q}, \omega, \chi)= & \frac{1}{64 \pi}\left\{\Theta(q-\omega) \sum_{\lambda= \pm} \lambda \Theta\left(\mu_{\chi}-\frac{q-\lambda \omega}{2}\right) G_{J}(q, \lambda \omega)-\Theta(\omega-q) \tilde{\Theta}\left(\frac{\omega+q}{2}-\mu_{\chi}\right)\right. \\
& \left.\Theta\left(\mu_{\chi}-\frac{\omega-q}{2}\right) G_{J}(-q,-\omega)\right\}, \\
& \operatorname{Re} F_{A}^{+}(\boldsymbol{q}, \omega, \chi)=\frac{\left(q^{2}-\omega^{2}\right) \mu_{\chi}}{8 \pi^{2} q^{2}}-\frac{1}{64 \pi^{2}} \sum_{\lambda, \lambda^{\prime}= \pm} G_{J}\left(\lambda q, \lambda^{\prime} \omega\right) H\left(\lambda q, \lambda^{\prime} \omega\right), \\
\operatorname{Im} F_{I}^{+}(\boldsymbol{q}, \omega, \chi)= & -\frac{1}{64 \pi}\left\{\Theta(q-\omega) \sum_{\lambda= \pm} \lambda \Theta\left(\mu-\frac{q-\lambda \omega}{2}\right) G_{I}(q, \lambda \omega)-\Theta(\omega-q)\right. \\
& \left.\times\left[\frac{8 \omega}{3} \Theta\left(\mu_{\chi}-\frac{\omega+q}{2}\right)+\tilde{\Theta}\left(\frac{\omega+q}{2}-\mu_{\chi}\right) \Theta\left(\mu_{\chi}-\frac{\omega-q}{2}\right) G_{I}(-q,-\omega)\right]\right\}, \\
& \operatorname{Re} F_{I}^{+}(\boldsymbol{q}, \omega, \chi)=-\frac{\omega \mu_{\chi}^{2}}{3 \pi^{2} q^{2}}+\frac{1}{64 \pi^{2}} \sum_{\lambda, \lambda^{\prime}= \pm} G_{I}\left(\lambda q, \lambda^{\prime} \omega\right) H\left(\lambda q, \lambda^{\prime} \omega\right),
\end{aligned}
$$

where these auxiliary functions are defined as

$$
\begin{aligned}
G_{f}(q, \xi) & =\frac{f\left(2 \mu_{\chi}+\xi\right)-f(q)}{q}, \\
H(q, \omega) & =\log \left|\frac{2 \mu_{\chi}+\omega-q}{\omega-q}\right|,
\end{aligned}
$$

with $f=S, M, N, J, I$ and $S(u)=2 u\left(u^{2}-3 q^{2}\right) / 3, M(u)=$ $\left(q^{2}-\omega^{2}\right)\left(3 q^{2}+u^{2}\right) u / 3 q^{2}, \quad N(u)=2 \omega^{2}\left(u^{2}-3 q^{2}\right) u / 3 q^{2}$, $J(u)=\left(q^{2}-\omega^{2}\right) u^{2} / q^{2}$, and $I(u)=2 \omega u\left(u^{2}-3 q^{2}\right) / 3 q^{2}$. $\Theta(x)$ is the Heaviside step function and the tilted Heaviside step function implies that $\tilde{\Theta}(x)=1$ for $x \geqslant 1$, and vanishes otherwise. $\Lambda$ is a cutoff wave vector relative to each Weyl node. Note that the density-density correlation function via the PVRS is the same as the one via other methods in Ref. [47]. Equations (16)-(35) are the central result of this paper.

The general correlation functions should obey several fundamental relations, such as the gauge invariance and the Kramers-Kronig relations. The gauge invariance (Ward identity) of the chirality-dependent correlation functions reads

$$
q_{\mu} \Pi^{\mu v}(\boldsymbol{q}, \omega, \chi)=0 .
$$

Inserting the expressions of correlation functions and using the relations $q_{0}=q^{0}=\omega, q_{\alpha}=-q^{\alpha}, q^{\alpha} q^{\alpha}=q_{\alpha} q_{\alpha}=q^{2}$, and $\delta_{\alpha \beta}=\delta^{\alpha \beta}$, one finds

$$
\begin{aligned}
& \omega \Pi^{00}(\boldsymbol{q}, \omega, \chi)-q^{2} F_{I}(\boldsymbol{q}, \omega, \chi)=0, \\
& {\left[\omega F_{I}(\boldsymbol{q}, \omega, \chi)-F_{L}(\boldsymbol{q}, \omega, \chi)\right] q^{\beta}=0 .}
\end{aligned}
$$

From Eq. (39), one immediately finds that the static density-current correlation functions vanish, $\Pi^{0 \alpha}(\boldsymbol{q}, 0, \chi)=$ $\Pi^{\alpha 0}(\boldsymbol{q}, 0, \chi)=0$. From Eq. (40), one has the relation $F_{L}(\boldsymbol{q}, 0, \chi)=0$.

It is straightforward to verify that the extrinsic part and the imaginary part of the intrinsic correlation functions satisfy the Ward identity. However, the nature of the cutoff scheme makes the intrinsic correlation function always contain some gauge-violating terms [60]. The intrinsic correlation function is usually written as

$$
\Pi_{-}^{\mu \nu}(\boldsymbol{q}, \omega, \chi)=\left[\left(\omega^{2}-q^{2}\right) g^{\mu \nu}-q^{\mu} q^{\nu}\right] \Pi(\boldsymbol{q}, \omega, \chi),
$$

where $g^{\mu v}=\operatorname{diag}(1,-1,-1,-1)$ is the metric tensor, and the overall scalar function $\Pi(\boldsymbol{q}, \omega, \chi)$ needs to be determined. From either the density-density correlation function via the PVRS in Eq. (17) or the results in Ref. [47], one finds

$$
\Pi_{-}^{00}(\boldsymbol{q}, \omega, \chi)=-\frac{q^{2}}{24 \pi^{2}} \log \frac{4 \Lambda^{2}}{q^{2}-\omega^{2}},
$$

which leads to the overall scalar function as

$$
\Pi(\boldsymbol{q}, \omega, \chi)=\frac{1}{24 \pi^{2}} \log \frac{4 \Lambda^{2}}{q^{2}-\omega^{2}} .
$$

In fact, this scalar function can be also extracted from the density-current correlation functions in Eqs. (14) and (25). To restore the gauge invariance of the intrinsic correlation function, we would like to subtract the nonlogarithmic terms in $\operatorname{Re} F_{T}^{-}$and $\operatorname{Re} F_{L}^{-}$and get

$$
\begin{gathered}
\operatorname{Re} F_{T}^{-}(\boldsymbol{q}, \omega, \chi)=\frac{\left(q^{2}-\omega^{2}\right)}{24 \pi^{2}} \log \left|\frac{4 \Lambda^{2}}{q^{2}-\omega^{2}}\right|, \\
\operatorname{Re} F_{L}^{-}(\boldsymbol{q}, \omega, \chi)=-\frac{\omega^{2}}{24 \pi^{2}} \log \left|\frac{4 \Lambda^{2}}{q^{2}-\omega^{2}}\right| .
\end{gathered}
$$

Let us consider the correlation functions for the interacting electrons. The simple way to encode the electron-electron interactions is the RPA. Within the RPA as illustrated in Fig. 1,

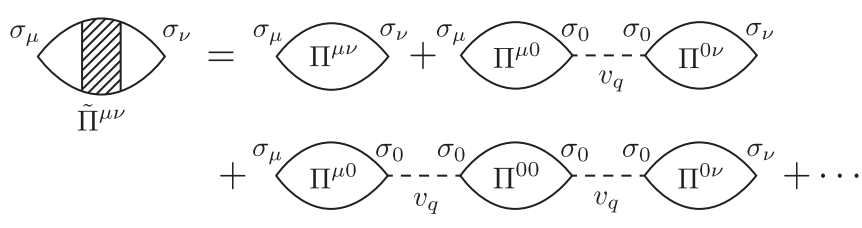

FIG. 1. Diagrammatic relation between the RPA correlation function $\tilde{\Pi}^{\mu v}(\boldsymbol{q}, \omega, \chi)$ and the noninteracting one $\Pi^{\mu v}(\boldsymbol{q}, \omega, \chi)$. The dashed line refers to the bare Coulomb interaction $v_{q}$. 
the correlation functions of the interacting 3D Weyl/Dirac semimetals can be expressed as

$$
\begin{aligned}
\tilde{\Pi}^{\mu v}(\boldsymbol{q}, \omega, \chi)= & \Pi^{\mu v}(\boldsymbol{q}, \omega, \chi) \\
& +\frac{\Pi^{\mu 0}(\boldsymbol{q}, \omega, \chi) v_{q} \Pi^{0 v}(\boldsymbol{q}, \omega, \chi)}{1-v_{q} \Pi^{00}(\boldsymbol{q}, \omega, \chi)},
\end{aligned}
$$

where $v_{q}=4 \pi e^{2} / \kappa q^{2}$ is the Fourier transform of the $3 \mathrm{D}$ Coulomb interaction and $\kappa$ is the effective dielectric constant.

For example, the interacting density-density response function with $\mu=v=0$ becomes

$$
\tilde{\Pi}^{00}(\boldsymbol{q}, \omega, \chi)=\frac{\Pi^{00}(\boldsymbol{q}, \omega, \chi)}{1-v_{q} \Pi^{00}(\boldsymbol{q}, \omega, \chi)},
$$

which recovers the widely used RPA density-density correlation function. On the other hand, the current-current correlation functions within the RPA are given as

$$
\begin{aligned}
\tilde{\Pi}^{\alpha \beta}(\boldsymbol{q}, \omega, \chi)= & \Pi^{\alpha \beta}(\boldsymbol{q}, \omega, \chi) \\
& +\frac{\Pi^{\alpha 0}(\boldsymbol{q}, \omega, \chi) v_{q} \Pi^{0 \beta}(\boldsymbol{q}, \omega, \chi)}{1-v_{q} \Pi^{00}(\boldsymbol{q}, \omega, \chi)} .
\end{aligned}
$$

It is clear that the nonvanishing density-current response functions give rise to corrections to the current-current correlation functions. Since $\Pi^{\alpha 0 / 0 \alpha}(\boldsymbol{q}, \omega, \chi)$ is parallel to the wave vector $q^{\alpha}$, there is no contribution to the antisymmetric part or the transverse part of the current-current correlation functions from the electron interactions. Note that for the 3D conventional electron gases in the absence of magnetic fields, the density-current response functions usually vanish. Thus the electron interaction does not renormalize the corresponding current-current correlation function within the RPA [38].

\section{GENERALIZED KRAMERS-KRONIG RELATIONS}

The Kramers-Kronig relation establishes a connection between the real and imaginary parts of the correlation functions and also allows us to verify the correctness of the correlation functions. Before generalizing the conventional KramersKronig relation to the one with $n$ th-order subtraction, let us first examine the causality relations among the correlation functions that enable us to find the negative-frequency part by taking the Hermitian conjugate of the positive-frequency part.

From the causality relations for chirality-dependent correlation functions (see Appendix D), one could write down the relations between the positive- and negative-frequency parts of each correlation function as follows:

$$
\begin{aligned}
\operatorname{Re} \Pi^{00}(\boldsymbol{q},-\omega, \chi) & =\operatorname{Re} \Pi^{00}(\boldsymbol{q}, \omega, \chi), \\
\operatorname{Im} \Pi^{00}(\boldsymbol{q},-\omega, \chi) & =-\operatorname{Im} \Pi^{00}(\boldsymbol{q}, \omega, \chi), \\
\operatorname{Re} F_{I}(\boldsymbol{q},-\omega, \chi) & =-\operatorname{Re} F_{I}(\boldsymbol{q}, \omega, \chi), \\
\operatorname{Im} F_{I}(\boldsymbol{q},-\omega, \chi) & =\operatorname{Im} F_{I}(\boldsymbol{q}, \omega, \chi), \\
\operatorname{Re} F_{X}(\boldsymbol{q},-\omega, \chi) & =\operatorname{Re} F_{X}(\boldsymbol{q}, \omega, \chi), \\
\operatorname{Im} F_{X}(\boldsymbol{q},-\omega, \chi) & =-\operatorname{Im} F_{X}(\boldsymbol{q}, \omega, \chi),
\end{aligned}
$$

where $X=T, L, A$. Based on all of the explicit expressions of chirality-dependent correlation functions in the previous section, it is instructive to crosscheck the real part from the imaginary part via the Kramers-Kronig relations or vice versa.

The conventional Kramers-Kronig relation for a complex function $f(\xi)$,

$$
f(\omega)=\frac{1}{\pi i} \mathcal{P} \int_{-\infty}^{\infty} d \xi \frac{f(\xi)}{\xi-\omega}+\mathcal{C}_{\infty},
$$

requires that the contribution from the semicircle at infinity $\mathcal{C}_{\infty}=C_{\infty}+i C_{\infty}^{\prime}$ tends to vanish, i.e., $C_{\infty}=0$ and $C_{\infty}^{\prime}=0$. Here $\mathcal{P}$ denotes the principal value of the integral. It is applicable to many causal response functions, such as the dielectric functions for the conventional electron gases [38] and 2D Dirac fermions [61]. Historically, Bjorken and Drell [62] had generalized Eq. (50) to a case with $\mathcal{C}_{\infty}$ being a complex constant that needs a first-order subtraction. Their generalization had been used to derive the correct density-density correlation function of 3D Weyl semimetals [47]. However, neither the conventional Kramers-Kronig relation nor Bjorken and Drell's generalization is adequate for the calculations of correlation functions of our interest. In this paper, we would like to provide a more general Kramers-Kronig relation with $n$ th-order subtraction, which is not only useful for our present calculations but also of great interest to many other physical problems.

If $f(\xi)$ does not diverge more than $\xi^{n-1}$ as $\xi \rightarrow \infty$, the generalized Kramers-Kronig relation is given as (detailed proof is presented in Appendix E)

$$
\begin{aligned}
f(\omega) \prod_{m=1}^{n} \frac{1}{\left(\omega-\omega_{m}\right)}= & \sum_{l=1}^{n} \frac{f\left(\omega_{l}\right)}{\left(\omega-\omega_{l}\right)} \prod_{m=1, m \neq l}^{n} \frac{1}{\left(\omega_{l}-\omega_{m}\right)} \\
& +\frac{1}{\pi i} \mathcal{P} \int_{-\infty}^{+\infty} d \xi \frac{f(\xi)}{g(\xi)}+\mathcal{C}_{\infty},
\end{aligned}
$$

where $g(\xi)=(\xi-\omega) \prod_{m=1}^{n}\left(\xi-\omega_{m}\right)$. The corresponding real and imaginary parts are given as

$$
\begin{aligned}
\operatorname{Re}[f(\omega)] \prod_{m=1}^{n} \frac{1}{\left(\omega-\omega_{m}\right)}= & \sum_{l=1}^{n} \frac{\operatorname{Re} f\left(\omega_{l}\right)}{\left(\omega-\omega_{l}\right)} \prod_{m=1, m \neq l}^{n} \frac{1}{\left(\omega_{l}-\omega_{m}\right)} \\
& +\frac{1}{\pi} \mathcal{P} \int_{-\infty}^{+\infty} d \xi \frac{\operatorname{Im} f(\xi)}{g(\xi)}+C_{\infty}, \\
\operatorname{Im}[f(\omega)] \prod_{m=1}^{n} \frac{1}{\left(\omega-\omega_{m}\right)}= & \sum_{l=1}^{n} \frac{\operatorname{Im} f\left(\omega_{l}\right)}{\left(\omega-\omega_{l}\right)} \prod_{m=1, m \neq l}^{n} \frac{1}{\left(\omega_{l}-\omega_{m}\right)} \\
& -\frac{1}{\pi} \mathcal{P} \int_{-\infty}^{+\infty} d \xi \frac{\operatorname{Re} f(\xi)}{g(\xi)}+C_{\infty}^{\prime},
\end{aligned}
$$

which ensure $\mathcal{C}_{\infty}$ vanishes and are named the Kramers-Kronig relations with $n$ th-order subtraction. It is one of the main results in this paper. In principle, the quantities of $\omega_{1}, \omega_{2}, \omega_{3}, \ldots$, and $\omega_{n}$ can be arbitrarily chosen as if it is not equal to $q$ in the real calculations. For the sake of simplicity, one could choose $\omega_{i}=$ $\alpha_{i} q$ for $i=1,2,3, \ldots, n$ with $\alpha_{1} \neq \alpha_{2} \neq \alpha_{3} \neq \cdots \neq \alpha_{n} \neq 1$. Note that the final result is independent of the specific values of $\omega_{i}$. 
TABLE I. The least order of subtraction $n$ in the generalized Kramers-Kronig relations for the correlation functions of 3D Weyl/Dirac semimetals. The index $+(-)$ refers to the extrinsic (intrinsic) part.

\begin{tabular}{lcccc}
\hline \hline Correlation functions & $\Pi_{+}^{\mu \nu}$ & $\Pi_{-}^{00}$ & $F_{A}^{-}, F_{I}^{-}$ & $F_{L}^{-}, F_{T}^{-}$ \\
\hline$n$ & 0 & 1 & 2 & 3 \\
\hline \hline
\end{tabular}

Let us explicitly verify the correlation functions by using the generalized Kramers-Kronig relations. The extrinsic part $F^{+}(\boldsymbol{q}, \xi, \chi) \rightarrow 0$ as $\xi \rightarrow \infty$, so that one needs to use only the conventional Kramers-Kronig relations in Eq. (50). However, since the intrinsic part $F^{-}(\boldsymbol{q}, \xi, \chi)$ does not vanish as $\xi \rightarrow \infty$, one must utilize the generalized Kramers-Kronig relations with $n$ th-order subtraction. The least orders of subtraction of the chirality-dependent correlation functions are tabulated in Table I. If the least order of subtraction is $m$, a higher-order subtraction $n>m$ would yield the same results except for more tedious mathematical manipulations.

To be specific, we take the intrinsic part of the densitycurrent correlation function as an example to demonstrate the application of the generalized Kramers-Kronig relations. We set $f(\xi)=F_{I}^{-}(\boldsymbol{q}, \xi, \chi)$ and have

$$
\operatorname{Im} f(\xi)=\frac{-\xi}{24 \pi} \Theta(\xi-q) .
$$

Since $\operatorname{Im} F_{I}^{-}(\boldsymbol{q}, \xi, \chi)$ does not diverge more than $\xi$ as $\xi \rightarrow \infty$, the generalized Kramers-Kronig relations with at least secondorder subtraction are needed to calculate $\operatorname{Re} F_{I}^{-}(\boldsymbol{q}, \omega, \chi)$ from $\operatorname{Im} F_{I}^{-}(\boldsymbol{q}, \omega, \chi)$. The Kramers-Kronig relations with secondorder subtraction has the form

$$
\begin{aligned}
\operatorname{Re} f(\omega)= & \frac{\left(\omega-\omega_{2}\right) \operatorname{Re} f\left(\omega_{1}\right)}{\left(\omega_{1}-\omega_{2}\right)}+\frac{\left(\omega-\omega_{1}\right) \operatorname{Re} f\left(\omega_{2}\right)}{\left(\omega_{2}-\omega_{1}\right)} \\
& +\frac{1}{\pi} \mathcal{P} \int_{-\infty}^{+\infty} d \xi \frac{\left(\omega-\omega_{1}\right)\left(\omega-\omega_{2}\right) \operatorname{Im} f(\xi)}{(\xi-\omega)\left(\xi-\omega_{1}\right)\left(\xi-\omega_{2}\right)} .
\end{aligned}
$$

Without loss of generality, we choose $\omega_{1}=\frac{1}{4} q$ and $\omega_{2}=\frac{1}{2} q$. After some straightforward calculations, we obtain

$$
\operatorname{Re} F_{I}^{-}(\boldsymbol{q}, \omega, \chi)=-\frac{\omega}{24 \pi^{2}} \log \left|\frac{4 \Lambda^{2}}{q^{2}-\omega^{2}}\right|,
$$

which is identical to the one in Eq. (25). Additionally, other functions involved in the correlation functions can be calculated in a similar way.

\section{THE OPTICAL CONDUCTIVITY}

In this section, the optical conductivity is obtained from the current-current correlation function in Weyl/Dirac semimetals. The general formula for the optical conductivity tensor is given as [39]

$$
\sigma^{\alpha \beta}(\omega)=\frac{i}{\omega+i \eta} \lim _{\boldsymbol{q} \rightarrow 0}\left[\Pi^{\alpha \beta}(\boldsymbol{q}, \omega)-\Pi^{\alpha \beta}(\boldsymbol{q}, 0)\right] .
$$

After taking the limit $\boldsymbol{q} \rightarrow 0$, the second term in the square bracket vanishes identically. The vanishing of the second term implies no diamagnetic current, similar to 2D Dirac fermions in graphene [63]. The real part of the optical conductivity $\sigma^{\alpha \beta}(\omega)$ is responsible for optical absorption and has the form

$$
\operatorname{Re} \sigma^{\alpha \beta}(\omega)=\sum_{\chi= \pm}\left[\frac{e^{2} \mu_{\chi}^{2}}{6 \pi v_{F} \hbar^{3}} \delta(\omega)+\frac{e^{2} \omega}{24 \pi v_{F} \hbar} \Theta\left(\hbar \omega-2\left|\mu_{\chi}\right|\right)\right] \delta^{\alpha \beta} .
$$

It is clear that the first term corresponds to the intraband part, while the second term is the interband part which onsets only above $2\left|\mu_{\chi}\right|$ with $\left(\mu_{\chi} \neq 0\right)$. When the Fermi level crosses the Weyl nodes $\mu_{\chi}=0$, the diagonal optical conductivity reduces to $\operatorname{Re} \sigma^{\alpha \alpha}(\omega)=\frac{e^{2} \omega}{24 \pi \hbar v_{F}} \Theta(\omega)$ [64-66]. Note that we have restored the factors of $\hbar, v_{F}$, and $e$ to make the physical units clear in the final equalities.

\section{DYNAMICAL CHIRAL MAGNETIC EFFECT}

The chiral magnetic conductivity, the antisymmetric part of the off-diagonal electronic conductivity tensor, of 3D Weyl fermions has been studied in some limits, such as the leading-order part [21] and the next-to-leading-order hard dense loop approximation $\left(\omega, q \ll\left|\mu_{\chi}\right|\right)$ [22]. Very recently, the dynamical CME or natural optical activity was revisited in the context of Weyl semimetals and metals without inversion symmetry from the semiclassical kinetic theory $[23,24]$. It has been pointed out that the dynamical CME has a geometric origin of Bloch bands and is directly related to the intrinsic magnetic moment of Bloch electrons on the Fermi surface. However, the semiclassical kinetic theory $[5,16,19,22-24]$ does not work well when the Fermi level is very close to the Weyl nodes, where the Berry curvature proportional to $1 / k^{2}$ tends to diverge as $k \rightarrow 0$ [5]. Meanwhile, the strong interband correlation at a tiny $k$ makes the single-band approximation in the semiclassical kinetic theory invalid. To comprehensively understand both the static and dynamical CMEs, one needs the exact current-current correlation functions, which allows us not only to reproduce the static and dynamical CMEs in some limits but also to obtain the corrections due to the higher-order spatial dispersion.

Within the linear response theory, the total current density induced by the optical field $\boldsymbol{A}(\mathbf{r}, t)=\boldsymbol{A}(\boldsymbol{q}, \omega) e^{i \boldsymbol{q} \cdot \mathbf{r}-i \omega t}$ can be written as

$$
j^{\alpha}(\boldsymbol{q}, \omega)=\Pi^{\alpha \beta}(\boldsymbol{q}, \omega) A^{\beta}(\boldsymbol{q}, \omega) .
$$

The Faraday's law, $\boldsymbol{B}(\boldsymbol{q}, \omega)=\boldsymbol{q} \times \boldsymbol{E}(\boldsymbol{q}, \omega) / \omega$, implies that a time-dependent magnetic field always comes together with a perpendicular electric field. Since the electric field is a vector and the magnetic field is a pseudovector, the CME coefficient is parity-odd. Due to the rotational and gauge invariance, one could adopt the chiral magnetic conductivity as [21]

$$
\sigma_{\mathrm{ch}}(\boldsymbol{q}, \omega)=\frac{1}{2 i q^{\alpha}} \varepsilon^{\alpha \beta \gamma} \Pi^{\beta \gamma}(\boldsymbol{q}, \omega),
$$

which only involves the antisymmetric part (or the parityodd part) of the current-current correlation function. Inserting 
the specific expression of current-current correlation function, $\Pi_{A}^{\alpha \beta}(\boldsymbol{q}, \omega)=\sum_{\chi= \pm} i \chi F_{A}(\boldsymbol{q}, \omega, \chi) \varepsilon^{\alpha \beta \gamma} q^{\gamma}$, immediately leads to

$$
\sigma_{\mathrm{ch}}(\boldsymbol{q}, \omega)=\sum_{\chi= \pm} \chi F_{A}(\boldsymbol{q}, \omega, \chi)
$$

It is nothing else but the exact chiral magnetic conductivity of 3D Weyl fermions.

Several remarks are in order here. First, $\sigma_{\mathrm{ch}}(\boldsymbol{q}, \omega)$ does not rely on those approximations made in Refs. [21-24]. Second, it should be emphasized that in the large chemical potential limit $\left|\mu_{\chi}\right| \gg q, \omega$, the chiral magnetic conductivity in Eq. (61) exactly recovers the previous result [22]. Third, $F_{A}(\boldsymbol{q}, \omega, \chi)$ is an odd function of the chirality-dependent chemical potential $\mu_{x}$ (see Appendix F). Finally, from the RPA procedure in Fig. 1, the electron interaction does not modify the antisymmetric part of current-current correlation functions such that the

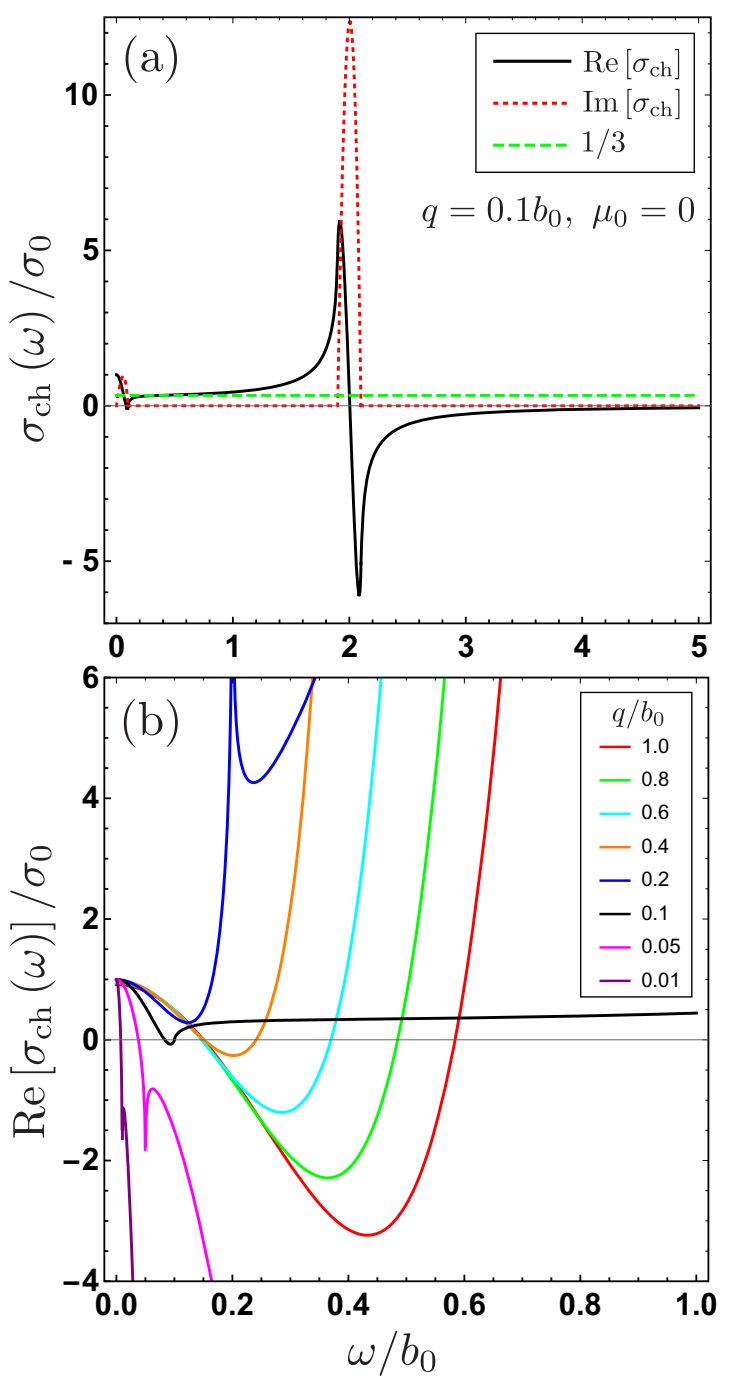

FIG. 2. The chiral magnetic conductivity as a function of frequency at zero temperature. (a) Real (black, solid) and imaginary (red, dotted) part of the chiral magnetic conductivity at $q=0.1 b_{0}, \mu_{0}=0$. The green dashed line indicates $1 / 3$. (b) The real part of the chiral magnetic conductivity for a set of values $q$. All the conductivities are scaled by $\sigma_{0}=e^{2} b_{0} / 2 \pi^{2}$. dynamical CME remains unchanged. Therefore, our results provide a more thorough understanding of the dynamical CME at nonzero frequencies and momentum. It is a second main result in this paper.

Figure 2(a) depicts the real and imaginary parts of the exact chiral magnetic conductivity at $q=0.1 b_{0}$ in Eq. (61) and captures all essential features of the chiral magnetic conductivity in Fig. 1 in Ref. [21]. First, one can clearly see a typical resonance behavior with a peak at $\omega=2 b_{0}$. Second, the chiral magnetic conductivity [black solid line in (a)] drops from $\sigma_{0}$ at $\omega=0$ to $\sigma_{0} / 3$. As shown in Fig. 2(b), the behavior of the chiral magnetic conductivity strongly depends on the magnitude of the wave vector $q$. The difference between the real part of the chiral magnetic conductivity in Eq. (61) and that in the hard dense loop approximation [22] is illustrated in Fig. 3. The approximate result in Ref. [22] is in good agreement with ours when $\omega /\left|\mu_{\chi}\right|$ or $q /\left|\mu_{\chi}\right|$ is less than 0.4 . However, the approximate chiral magnetic conductivity shows a noticeable deviation from our exact one when the ratio of $\omega /\left|\mu_{\chi}\right|$ or $q /\left|\mu_{\chi}\right|$ starts to exceed 0.6.

There are two distinct limits for the chiral magnetic conductivity: the static limit ( $\omega=0$ before $\boldsymbol{q} \rightarrow 0$ ) and the uniform limit $(\boldsymbol{q}=0$ before $\omega \rightarrow 0$ ) [39]. Let us first examine the static limit,

$$
\lim _{\boldsymbol{q} \rightarrow 0} \lim _{\omega \rightarrow 0} \operatorname{Re} F_{A}(\boldsymbol{q}, \omega, \chi)=\frac{e^{2} \mu_{\chi}}{4 \pi^{2}} .
$$

Thus the chiral magnetic conductivity for a pair of Weyl nodes in the static limit becomes

$$
\sigma_{\mathrm{ch}}(\boldsymbol{q}, \omega)=\frac{e^{2} b_{0}}{2 \pi^{2} \hbar^{2} c} .
$$

If $b_{0}$ is regarded as the chiral chemical potential induced by the parallel electric and magnetic fields via the chiral anomaly [47], the static chiral magnetic conductivity vanishes identically and

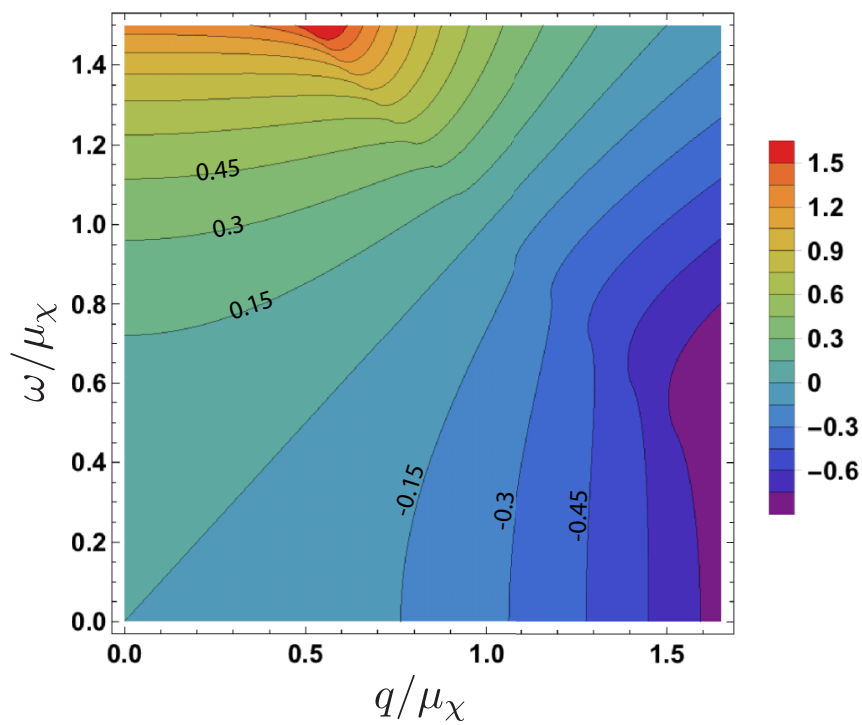

FIG. 3. Contour plot of the difference between the real part of the chiral magnetic conductivity of one Weyl node in Eq. (61) and that of Eq. (84) in Ref. [22] at zero temperature. The results are normalized to $e^{2} \mu_{\chi} / 12 \pi^{2}$. 
agrees with the general semiclassical analysis [16] and the numerical simulations in the lattice models $[17,19]$. On the other hand, if $b_{0}$ is the energy difference of Weyl nodes due to the inversion symmetry breaking, one may naively expect a nonzero electric current induced by a static magnetic field, which is unfortunately inconsistent with the fact that there is no equilibrium current in solids in the static limit [39]. The controversy of the chiral magnetic conductivity might be resolved by introducing the Bardeen-Zumino Chern-Simons term [18,67-70].

Similarly, one evaluates the uniform limit and obtains

$$
\lim _{\omega \rightarrow 0} \lim _{\boldsymbol{q} \rightarrow 0} \operatorname{Re} F_{A}(\boldsymbol{q}, \omega, \chi)=\frac{e^{2} \mu_{\chi}}{12 \pi^{2}}
$$

which gives rise to the corresponding chiral magnetic conductivity in the uniform limit

$$
\sigma_{\mathrm{ch}}(\boldsymbol{q}, \omega)=\frac{e^{2} b_{0}}{6 \pi^{2} \hbar^{2} c},
$$

where $b_{0}$ refers to the energy difference of Weyl nodes with opposite chirality. Our result here is consistent with those of Refs. [21-24].

To gain more insights into the dynamical CME, we consider the limit that the Fermi energy is far from the Weyl nodes $\left|\mu_{\chi}\right| \gg \omega, q$. Expanding $\operatorname{Re} F_{A}(\boldsymbol{q}, \omega, \chi)$ in power of $\boldsymbol{q}$ or $\omega$ and keeping the correction up to $\mathcal{O}\left(q^{3}\right)$ or $\mathcal{O}\left(\omega^{3}\right)$, one finds the real part

$$
\operatorname{Re} F_{A}(\boldsymbol{q}, \omega, \chi) \approx \begin{cases}\frac{e^{2} \mu_{\chi}}{12 \pi^{2}}\left(1-\frac{2 q^{2}}{5 \omega^{2}}\right), & \omega \gg q ; \\ \frac{e^{2} \mu_{\chi}}{4 \pi^{2}}\left(1-\frac{2 \omega^{2}}{q^{2}}\right), & q \gg \omega .\end{cases}
$$

The first term in each line on the right-hand side corresponds to the chiral magnetic conductivities in two different limits (uniform and static), while the second term is the new leadingorder correction. In sum, the exact chiral magnetic conductivity in Eq. (61) possesses more rich features than the two distinct limits.

\section{MAGNETIC SUSCEPTIBILITIES}

In this section, we first consider the Pauli susceptibility and the orbital magnetic susceptibility of the noninteracting 3D Weyl/Dirac semimetals in the weak-magnetic-field limit, thus neglecting the Landau-level structure of Weyl nodes. We then briefly discuss the impact of electron interactions on these magnetic susceptibilities within the RPA.

The Pauli spin susceptibility of noninteracting electrons follows from the limit

$$
\chi_{P}^{0}=-\left(\frac{g \mu_{B}}{2}\right)^{2} \lim _{q \rightarrow 0} \sum_{\chi= \pm} \Pi^{z z}(q \hat{z}, 0, \chi),
$$

where $\mu_{B}$ is the Bohr magneton of free electrons and $g$ is the material-dependent $g$ factor.

If the Pauli matrices $\sigma^{i}$ in Eq. (1) refer to the pseudospin degree of freedom (3D analogs of graphene), the spin response function of a noninteracting system equals the density-density susceptibility,

$$
\begin{aligned}
\chi_{P}^{0} & =-\left(\frac{g \mu_{B}}{2}\right)^{2} \lim _{q \rightarrow 0} \sum_{\chi= \pm} \Pi^{00}(q, 0, \chi) \\
& =\left(\frac{g \mu_{B}}{2}\right)^{2} \sum_{\chi= \pm} N\left(\mu_{\chi}\right),
\end{aligned}
$$

which is proportional to the sum of the density of states near the Fermi level $N\left(\mu_{\chi}\right)=\mu_{\chi}^{2} /\left(2 \pi^{2} v_{F}^{3} \hbar^{3}\right)$, similar to the conventional electron gases and graphene [38,61].

On the other hand, if the Pauli matrices $\sigma^{i}$ in Eq. (1) refer to the real spin of electrons, the spin response function of a noninteracting system vanishes identically,

$$
\chi_{P}^{0}=-\left(\frac{g \mu_{B}}{2}\right)^{2} \lim _{q \rightarrow 0} \sum_{\chi= \pm} F_{L}(q, 0, \chi)=0,
$$

which can be traced back to the fact that the spin-momentum locking causes the average Zeeman energy over the Fermi surface near each Weyl node to vanish. It is worth noting that the vanishing of the Pauli spin susceptibilities had been calculated in the Landau-level basis [71] and possibly observed in NbAs, a candidate of Weyl semimetal, at the quantum limit [72].

For 3D Weyl/Dirac semimetals, a magnetic field usually produces both the orbital diamagnetism and the splitting of Weyl nodes with opposite chirality in momentum space $\boldsymbol{b} \neq$ 0 through the orbital motion of electrons and the Zeeman interaction, respectively. The former corresponds to the orbital magnetic susceptibility, while the latter leads to a finite orbital magnetization [5]. The transverse current-current correlation function allows us to calculate the noninteracting orbital magnetic susceptibility induced by a static magnetic field,

$$
\begin{aligned}
\chi_{\mathrm{orb}}^{0} & =-\frac{e^{2} v_{F}^{2}}{c^{2}} \sum_{\chi= \pm} \lim _{q \rightarrow 0} \frac{\Pi^{z z}(q \hat{x}, 0, \chi)}{q^{2}} \\
& =\frac{-e^{2} v_{F}}{12 \pi^{2} \hbar c^{2}} \log \frac{\varepsilon_{c}^{2}}{\left|\mu_{+} \mu_{-}\right|}+\frac{5 e^{2} v_{F}}{36 \pi^{2} \hbar c^{2}},
\end{aligned}
$$

where $\varepsilon_{c}=v_{F} \hbar \Lambda$ is the cutoff energy measured from each Weyl node. The first term in the second line exactly agrees with the one obtained from the thermodynamic potential in the quantum limit [71]. However, the second term was absent there. Since the cutoff energy $\varepsilon_{c}$ is far greater than the chirality-dependent chemical potential $\left|\mu_{\chi}\right|$, the second term becomes negligible, leading to an orbital diamagnetism. This logarithmic divergence of the orbital diamagnetism was attributed to the relativistic nature of 3D Weyl/Dirac fermions.

According to the semiclassical formula of the orbital magnetization [5], the orbital magnetization for a pair of Weyl nodes with a finite momentum spacing $\boldsymbol{b}=(0,0, b)$ is given as

$$
M_{z}=-\frac{\mu_{0}}{e c} \sigma_{x y}=\frac{e \mu_{0} b}{2 \pi^{2} \hbar c},
$$

where $\sigma_{x y}=-\frac{e^{2} b}{2 \pi^{2} \hbar}$ is the corresponding anomalous Hall conductivity at $\mu_{0}=0$ [73] and $b=g \mu_{B} B$. Our semiclassical estimation of the orbital magnetization is consistent with the spin-orbit component from the direct derivative of the thermodynamic potential with respect to the orbital part and the spin part of magnetic fields [71]. This part of orbital 


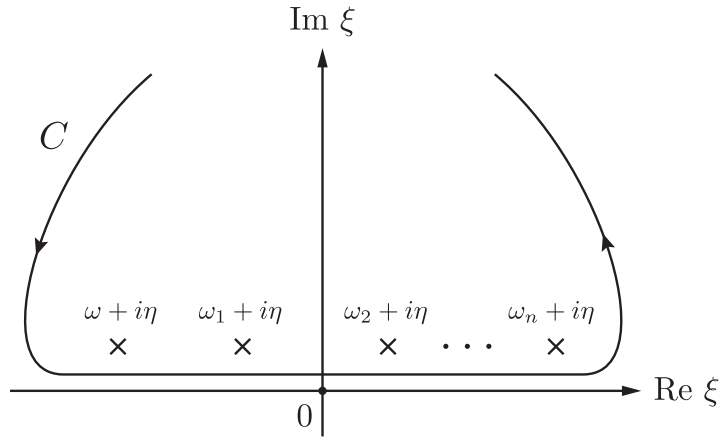

FIG. 4. Contour in the upper half $\xi$ plane for Cauchy integral with $n+1$ poles in Eq. (E1).

magnetization is related to the Fermi arc states that connect the two Weyl nodes. It is well known that the Pauli susceptibility and the orbital magnetic susceptibility of the 3D noninteracting electron gases with a single parabolic band satisfy the relation $\chi_{P}^{0}=-3 \chi_{\text {orb }}^{0}$ [38]. It is clear that the ratio between the orbital and Pauli susceptibilities of 3D Weyl fermions greatly deviates from $-1 / 3$ of the $3 \mathrm{D}$ electron gases.

Before closing this section, let us briefly discuss the impact of electron interactions on the magnetic susceptibilities. Following the RPA procedure in Fig. 1, one finds that there is no correction to either the Pauli susceptibility or the orbital magnetic susceptibility from electron interactions. In addition, another RPA procedure was proposed to compute the orbital magnetic susceptibility in the context of graphene [75,76]:

$$
\tilde{\chi}_{\text {orb }}=-\frac{e^{2} v_{F}^{2}}{c^{2}} \lim _{q \rightarrow 0} \frac{\Pi^{z z}(q \hat{x}, 0)}{q^{2}\left[1-v_{q} \Pi^{z z}(q \hat{x}, 0)\right]} .
$$

Within this RPA procedure, the orbital magnetic susceptibility of 3D interacting Weyl fermions becomes

$$
\tilde{\chi}_{\text {orb }}=\sum_{\chi= \pm} \frac{\chi_{\text {orb }}^{0}(\chi)}{\left[1-\frac{4 \pi e^{2}}{\kappa} \cdot \frac{1}{12 \pi^{2} v_{F} \hbar}\left(\log \frac{\varepsilon_{c}}{\left|\mu_{\chi}\right|}-\frac{5}{6}\right)\right]},
$$

where $\chi_{\mathrm{orb}}^{0}(\chi)$ refers to the orbital magnetic susceptibilities of noninteracting Weyl fermions of chirality $\chi$ in Eq. (70). One can see that the interaction effect gives rise to a $\Lambda$-dependent renormalization of the orbital magnetic susceptibilities. For a sufficiently large ratio $\varepsilon_{c} /\left|\mu_{\chi}\right|$, the interacting orbital magnetic susceptibility $\tilde{\chi}_{\text {orb }}$ might change a sign. In other words, a transition from the orbital diamagnetism to the orbital paramagnetism could occur through tuning the Fermi energy or the carrier concentration. The interacting susceptibility for the orbital paramagnetism becomes

$$
\tilde{\chi}_{\text {orb }}=\frac{\kappa v_{F}^{2}}{2 \pi c^{2}},
$$

which turns out to be independent of the cutoff $\Lambda$. One should bear in mind that when the Fermi level approaches the Weyl nodes, the carrier density becomes very low, such that the RPA might become invalid. Thus, the interaction-driven transition of orbital magnetism requires a more sophisticated treatment beyond the simple RPA, such as the higher-order perturbation theory and the GW approximation [38].

\section{CONCLUSIONS AND DISCUSSIONS}

In summary, the dynamical correlation functions of $3 \mathrm{D}$ Weyl/Dirac semimetals have been derived analytically via the PVRS at zero temperature. The gauge invariance and Kramers-Kronig relations among these correlation functions have been verified in detail. We have obtained the exact chiral magnetic conductivity and deepened the understanding of the CME. We have calculated the magnetic susceptibilities as well as the orbital magnetization. The impacts of electron interactions within the RPA on the magnetic susceptibilities are also discussed. In addition, the dynamical correlation functions might be useful to explore the nonlocal transport and optical properties due to the higher-order spatial dispersion of $3 \mathrm{D}$ Weyl/Dirac semimetals in the presence of time- and spatially varying external fields, such as Lorentz birefringence and Jones birefringence [77].

Note added. Recently an independent study [78] appeared which derives the diagonal current-current correlation functions by a different approach and computes some related quantities.

\section{ACKNOWLEDGMENTS}

We are grateful to Wen-Yu Shan for useful discussions and to Shi-Xiong Wang for preparing the figures and a careful reading of the manuscript. H.-R.C. was supported by the National Natural Science Foundation of China under Grant No. 11547200, the China Scholarship Council (No. 201608515061), the NSERC of Canada, and FQRNT of Quebec (Hong Guo). J.Z. was supported by the Research Grant Council, University Grants Committee, Hong Kong, under Grants No. 17301116 and No. C6026-16W. J.Z. also acknowledges the hospitality of the Department of Physics at Southern University of Science and Technology. H.-R.C. would like to dedicate this paper to the memory of Professor Mu-Lin Yan.

\section{APPENDIX A: DECOMPOSITION OF THE CORRELATION FUNCTIONS VIA PVRS}

To apply the PVRS to evaluate the correlation functions contains two steps. The first step is to reduce the correlation functions to six basic tensor integrals (including two basic scalar integrals). The second is to further decompose them into seven basic scalar integrals by utilizing PVRS.

By utilizing the traces of products of Pauli matrices,

$$
\begin{aligned}
\operatorname{Tr}\left[\sigma^{\mu}\right] & =2 \delta^{\mu 0}, \\
\operatorname{Tr}\left[\sigma^{\alpha} \sigma^{\beta}\right] & =2 \delta^{\alpha \beta}, \\
\operatorname{Tr}\left[\sigma^{\alpha} \sigma^{\beta} \sigma^{\tau}\right] & =2 i \varepsilon^{\alpha \beta \tau}, \\
\operatorname{Tr}\left[\sigma^{\alpha} \sigma^{\tau} \sigma^{\beta} \sigma^{\rho}\right] & =2\left(\delta^{\alpha \tau} \delta^{\beta \rho}-\delta^{\alpha \beta} \delta^{\tau \rho}+\delta^{\alpha \rho} \delta^{\beta \tau}\right),
\end{aligned}
$$


we decompose the four chirality-dependent correlation functions as follows:

$$
\begin{gathered}
\Pi^{00}\left(\boldsymbol{q}, i \omega_{m}, \chi\right)=\frac{1}{2}\left[A_{0}+\delta^{\tau \rho}\left(B^{\tau \rho}+B^{\tau} q^{\rho}\right)\right], \\
\Pi^{\alpha \beta}\left(\boldsymbol{q}, i \omega_{m}, \chi\right)=\frac{1}{2}\left[\delta^{\alpha \beta} A_{0}+\left(\delta^{\alpha \tau} \delta^{\beta \rho}-\delta^{\alpha \beta} \delta^{\tau \rho}+\delta^{\alpha \rho} \delta^{\beta \tau}\right)\left(B^{\tau \rho}+B^{\tau} q^{\rho}\right)+i \chi \varepsilon^{\alpha \beta \tau}\left(C^{\tau}+C_{0} q^{\tau}-D^{\tau}\right)\right], \\
\Pi^{0 \alpha}\left(\boldsymbol{q}, i \omega_{m}, \chi\right)=\frac{1}{2}\left[\delta^{\alpha \tau}\left(C^{\tau}+C_{0} q^{\tau}\right)+\delta^{\alpha \tau} D_{\tau}+\chi i \varepsilon^{\tau \alpha \rho}\left(B^{\tau \rho}+B^{\tau} q^{\rho}\right)\right], \\
\Pi^{\alpha 0}\left(\boldsymbol{q}, i \omega_{m}, \chi\right)=\frac{1}{2}\left[\delta^{\alpha \tau}\left(C^{\tau}+C_{0} q^{\tau}\right)+\delta^{\alpha \tau} D^{\tau}+\chi i \varepsilon^{\alpha \tau \rho}\left(B^{\tau \rho}+B^{\tau} q^{\rho}\right)\right],
\end{gathered}
$$

which clearly shows that the four chirality-dependent correlation functions are reduced to six integrals,

$$
\begin{aligned}
& A_{0}\left(\boldsymbol{q}, i \omega_{m}, \chi\right) \equiv \frac{1}{\mathcal{V}} \sum_{\boldsymbol{k}} \frac{1}{\beta_{T}} \sum_{i \Omega_{n}} \sum_{\lambda, \lambda^{\prime}= \pm} \frac{1}{i \Omega_{n}+\mu_{\chi}-\varepsilon_{\lambda}(\boldsymbol{k})} \frac{1}{i \Omega_{n}+i \omega_{m}+\mu_{\chi}-\varepsilon_{\lambda^{\prime}}(\boldsymbol{k}+\boldsymbol{q})}, \\
& B^{\tau \rho}\left(\boldsymbol{q}, i \omega_{m}, \chi\right) \equiv \frac{1}{\mathcal{V}} \sum_{\boldsymbol{k}} \frac{1}{\beta_{T}} \sum_{i \Omega_{n}} \sum_{\lambda, \lambda^{\prime}= \pm} \lambda \lambda^{\prime} \frac{k^{\tau} k^{\rho}}{|\boldsymbol{k}||\boldsymbol{k}+\boldsymbol{q}|} \frac{1}{i \Omega_{n}+\mu_{\chi}-\varepsilon_{\lambda}(\boldsymbol{k})} \frac{1}{i \Omega_{n}+i \omega_{m}+\mu_{\chi}-\varepsilon_{\lambda^{\prime}}(\boldsymbol{k}+\boldsymbol{q})}, \\
& B^{\tau}\left(\boldsymbol{q}, i \omega_{m}, \chi\right) \equiv \frac{1}{\mathcal{V}} \sum_{\boldsymbol{k}} \frac{1}{\beta_{T}} \sum_{i \Omega_{n}} \sum_{\lambda, \lambda^{\prime}= \pm} \lambda \lambda^{\prime} \frac{k^{\tau}}{|\boldsymbol{k}||\boldsymbol{k}+\boldsymbol{q}|} \frac{1}{i \Omega_{n}+\mu_{\chi}-\varepsilon_{\lambda}(\boldsymbol{k})} \frac{1}{i \Omega_{n}+i \omega_{m}+\mu_{\chi}-\varepsilon_{\lambda^{\prime}}(\boldsymbol{k}+\boldsymbol{q})}, \\
& C^{\tau}\left(\boldsymbol{q}, i \omega_{m}, \chi\right) \equiv \frac{1}{\mathcal{V}} \sum_{\boldsymbol{k}} \frac{1}{\beta_{T}} \sum_{i \Omega_{n}} \sum_{\lambda, \lambda^{\prime}= \pm} \lambda^{\prime} \frac{k^{\tau}}{|\boldsymbol{k}+\boldsymbol{q}|} \frac{1}{i \Omega_{n}+\mu_{\chi}-\varepsilon_{\lambda}(\boldsymbol{k})} \frac{1}{i \Omega_{n}+i \omega_{m}+\mu_{\chi}-\varepsilon_{\lambda^{\prime}}(\boldsymbol{k}+\boldsymbol{q})}, \\
& C_{0}\left(\boldsymbol{q}, i \omega_{m}, \chi\right) \equiv \frac{1}{\mathcal{V}} \sum_{\boldsymbol{k}} \frac{1}{\beta_{T}} \sum_{i \Omega_{n}} \sum_{\lambda, \lambda^{\prime}= \pm} \lambda^{\prime} \frac{1}{|\boldsymbol{k}+\boldsymbol{q}|} \frac{1}{i \Omega_{n}+\mu_{\chi}-\varepsilon_{\lambda}(\boldsymbol{k})} \frac{1}{i \Omega_{n}+i \omega_{m}+\mu_{\chi}-\varepsilon_{\lambda^{\prime}}(\boldsymbol{k}+\boldsymbol{q})}, \\
& D^{\tau}\left(\boldsymbol{q}, i \omega_{m}, \chi\right) \equiv \frac{1}{\mathcal{V}} \sum_{\boldsymbol{k}} \frac{1}{\beta_{T}} \sum_{i \Omega_{n}} \sum_{\lambda, \lambda^{\prime}= \pm} \lambda \frac{k^{\tau}}{|\boldsymbol{k}|} \frac{1}{i \Omega_{n}+\mu_{\chi}-\varepsilon_{\lambda}(\boldsymbol{k})} \frac{1}{i \Omega_{n}+i \omega_{m}+\mu_{\chi}-\varepsilon_{\lambda^{\prime}}(\boldsymbol{k}+\boldsymbol{q})} .
\end{aligned}
$$

Here $A_{0}\left(\boldsymbol{q}, i \omega_{m}, \chi\right)$ and $C_{0}\left(\boldsymbol{q}, i \omega_{m}, \chi\right)$ are scalar integrals and relatively easy to be evaluated. Our main task is to compute the complicated tensor integrals $B^{\tau \rho}\left(\boldsymbol{q}, i \omega_{m}, \chi\right), B^{\tau}\left(\boldsymbol{q}, i \omega_{m}, \chi\right), C^{\tau}\left(\boldsymbol{q}, i \omega_{m}, \chi\right)$, and $D^{\tau}\left(\boldsymbol{q}, i \omega_{m}, \chi\right)$ via PVRS. Using the ansatz

$$
\begin{gathered}
B^{\tau}\left(\boldsymbol{q}, i \omega_{m}, \chi\right) \equiv B_{1}\left(\boldsymbol{q}, i \omega_{m}, \chi\right) q^{\tau}, \\
B^{\tau \rho}\left(\boldsymbol{q}, i \omega_{m}, \chi\right) \equiv B_{00}\left(\boldsymbol{q}, i \omega_{m}, \chi\right) \delta^{\tau \rho}+B_{11}\left(\boldsymbol{q}, i \omega_{m}, \chi\right) q^{\tau} q^{\rho}, \\
C^{\tau}\left(\boldsymbol{q}, i \omega_{m}, \chi\right) \equiv C_{1}\left(\boldsymbol{q}, i \omega_{m}, \chi\right) q^{\tau} \\
D^{\tau}\left(\boldsymbol{q}, i \omega_{m}, \chi\right) \equiv D_{1}\left(\boldsymbol{q}, i \omega_{m}, \chi\right) q^{\tau},
\end{gathered}
$$

we have

$$
\begin{gathered}
B^{\tau}\left(\boldsymbol{q}, i \omega_{m}, \chi\right) q^{\tau} \equiv B_{1}\left(\boldsymbol{q}, i \omega_{m}, \chi\right) q^{\tau} q^{\tau}=B_{1}\left(\boldsymbol{q}, i \omega_{m}, \chi\right) q^{2}, \\
C^{\tau}\left(\boldsymbol{q}, i \omega_{m}, \chi\right) q^{\tau} \equiv C_{1}\left(\boldsymbol{q}, i \omega_{m}, \chi\right) q^{\tau} q^{\tau}=C_{1}\left(\boldsymbol{q}, i \omega_{m}, \chi\right) q^{2}, \\
D^{\tau}\left(\boldsymbol{q}, i \omega_{m}, \chi\right) q^{\tau} \equiv D_{1}\left(\boldsymbol{q}, i \omega_{m}, \chi\right) q^{\tau} q^{\tau}=D_{1}\left(\boldsymbol{q}, i \omega_{m}, \chi\right) q^{2}, \\
B^{\tau \rho}\left(\boldsymbol{q}, i \omega_{m}, \chi\right) \delta^{\tau \rho} \equiv B_{00}\left(\boldsymbol{q}, i \omega_{m}, \chi\right) \delta^{\tau \rho} \delta^{\tau \rho}+B_{11}\left(\boldsymbol{q}, i \omega_{m}, \chi\right) q^{\tau} q^{\rho} \delta^{\tau \rho} \\
=3 B_{00}\left(\boldsymbol{q}, i \omega_{m}, \chi\right)+q^{2} B_{11}\left(\boldsymbol{q}, i \omega_{m}, \chi\right) \equiv B_{a}\left(\boldsymbol{q}, i \omega_{m}, \chi\right), \\
B^{\tau \rho}\left(\boldsymbol{q}, i \omega_{m}, \chi\right) q^{\tau} q^{\rho} \equiv B_{00}\left(\boldsymbol{q}, i \omega_{m}, \chi\right) \delta^{\tau \rho} q^{\tau} q^{\rho}+B_{11}\left(\boldsymbol{q}, i \omega_{m}, \chi\right) q^{\tau} q^{\rho} q^{\tau} q^{\rho} \\
=B_{00}\left(\boldsymbol{q}, i \omega_{m}, \chi\right) q^{2}+B_{11}\left(\boldsymbol{q}, i \omega_{m}, \chi\right)\left(q^{2}\right)^{2} \equiv q^{2} B_{b}\left(\boldsymbol{q}, i \omega_{m}, \chi\right),
\end{gathered}
$$

where we have summed over the repeated indices and applied the relations $\delta^{\tau \rho} \delta^{\tau \rho}=3$ and $q^{\tau} q^{\tau}=\boldsymbol{q}^{2}=q^{2}$. Solving $B_{00}\left(\boldsymbol{q}, i \omega_{m}, \chi\right)$ and $B_{11}\left(\boldsymbol{q}, i \omega_{m}, \chi\right)$ in the last two equations in terms of $B_{a}\left(\boldsymbol{q}, i \omega_{m}, \chi\right)$ and $B_{b}\left(\boldsymbol{q}, i \omega_{m}, \chi\right)$ leads to

$$
\begin{aligned}
B_{00}\left(\boldsymbol{q}, i \omega_{m}, \chi\right) & \equiv \frac{B_{a}\left(\boldsymbol{q}, i \omega_{m}, \chi\right)-B_{b}\left(\boldsymbol{q}, i \omega_{m}, \chi\right)}{2}, \\
B_{11}\left(\boldsymbol{q}, i \omega_{m}, \chi\right) & \equiv \frac{3 B_{b}\left(\boldsymbol{q}, i \omega_{m}, \chi\right)-B_{a}\left(\boldsymbol{q}, i \omega_{m}, \chi\right)}{2 q^{2}} .
\end{aligned}
$$


The tensor integrals $B^{\tau \rho}\left(\boldsymbol{q}, i \omega_{m}, \chi\right), B^{\tau}\left(\boldsymbol{q}, i \omega_{m}, \chi\right), C^{\tau}\left(\boldsymbol{q}, i \omega_{m}, \chi\right)$, and $D^{\tau}\left(\boldsymbol{q}, i \omega_{m}, \chi\right)$ can be expressed in terms of the following five scalar integrals $B_{a}, B_{b}, B_{1}, C_{1}$, and $D_{1}$ :

$$
\begin{gathered}
B_{a}\left(\boldsymbol{q}, i \omega_{m}, \chi\right) \equiv \frac{1}{\mathcal{V}} \sum_{\boldsymbol{k}} \frac{1}{\beta_{T}} \sum_{i \Omega_{n}} \sum_{\lambda, \lambda^{\prime}= \pm} \lambda \lambda^{\prime} \frac{|\boldsymbol{k}|}{|\boldsymbol{k}+\boldsymbol{q}|} \frac{1}{i \Omega_{n}+\mu_{\chi}-\varepsilon_{\lambda}(\boldsymbol{k})} \frac{1}{i \Omega_{n}+i \omega_{m}+\mu_{\chi}-\varepsilon_{\lambda^{\prime}}(\boldsymbol{k}+\boldsymbol{q})}, \\
q^{2} B_{b}\left(\boldsymbol{q}, i \omega_{m}, \chi\right) \equiv \frac{1}{\mathcal{V}} \sum_{\boldsymbol{k}} \frac{1}{\beta_{T}} \sum_{i \Omega_{n}} \sum_{\lambda, \lambda^{\prime}= \pm} \lambda \lambda^{\prime} \frac{(\boldsymbol{k} \cdot \boldsymbol{q})^{2}}{|\boldsymbol{k}||\boldsymbol{k}+\boldsymbol{q}|} \frac{1}{i \Omega_{n}+\mu_{\chi}-\varepsilon_{\lambda}(\boldsymbol{k})} \frac{1}{i \Omega_{n}+i \omega_{m}+\mu_{\chi}-\varepsilon_{\lambda^{\prime}}(\boldsymbol{k}+\boldsymbol{q})}, \\
q^{2} B_{1}\left(\boldsymbol{q}, i \omega_{m}, \chi\right) \equiv \frac{1}{\mathcal{V}} \sum_{\boldsymbol{k}} \frac{1}{\beta_{T}} \sum_{i \Omega_{n}} \sum_{\lambda, \lambda^{\prime}= \pm} \lambda \lambda^{\prime} \frac{\boldsymbol{k} \cdot \boldsymbol{q}}{|\boldsymbol{k}||\boldsymbol{k}+\boldsymbol{q}|} \frac{1}{i \Omega_{n}+\mu_{\chi}-\varepsilon_{\lambda}(\boldsymbol{k})} \frac{1}{i \Omega_{n}+i \omega_{m}+\mu_{\chi}-\varepsilon_{\lambda^{\prime}}(\boldsymbol{k}+\boldsymbol{q})}, \\
q^{2} C_{1}\left(\boldsymbol{q}, i \omega_{m}, \chi\right) \equiv \frac{1}{\mathcal{V}} \sum_{\boldsymbol{k}} \frac{1}{\beta_{T}} \sum_{i \Omega_{n}} \sum_{\lambda, \lambda^{\prime}= \pm} \lambda^{\prime} \frac{1}{|\boldsymbol{k}+\boldsymbol{q}|} \frac{1}{i \Omega_{n}+\mu_{\chi}-\varepsilon_{\lambda}(\boldsymbol{k})} \frac{1}{i \Omega_{n}+i \omega_{m}+\mu_{\chi}-\varepsilon_{\lambda^{\prime}}(\boldsymbol{k}+\boldsymbol{q})}, \\
q^{2} D_{1}\left(\boldsymbol{q}, i \omega_{m}, \chi\right) \equiv \frac{1}{\mathcal{V}} \sum_{\boldsymbol{k}} \frac{1}{\beta_{T}} \sum_{i \Omega_{n}} \sum_{\lambda, \lambda^{\prime}= \pm} \frac{1}{\lambda \frac{k_{\tau}}{|\boldsymbol{k}|} \frac{1}{i \Omega_{n}+\mu_{\chi}-\varepsilon_{\lambda}(\boldsymbol{k})} \frac{1}{i \Omega_{n}+i \omega_{m}+\mu_{\chi}-\varepsilon_{\lambda^{\prime}}(\boldsymbol{k}+\boldsymbol{q})} .}
\end{gathered}
$$

Summing over Matsubara frequency $\Omega_{n}$ and performing analytical continuum $i \omega_{m} \rightarrow \omega+i \eta$, one can obtain the following seven scalar functions:

$$
\begin{gathered}
A_{0}(\boldsymbol{q}, \omega, \chi)=\frac{1}{\mathcal{V}} \sum_{\boldsymbol{k}} \sum_{\lambda, \lambda^{\prime}= \pm} \mathcal{F}_{\lambda \lambda^{\prime}}(\boldsymbol{k}, \boldsymbol{k}+\boldsymbol{q}, \omega), \\
q^{2} B_{1}(\boldsymbol{q}, \omega, \chi)=\frac{1}{\mathcal{V}} \sum_{\boldsymbol{k}} \frac{(\boldsymbol{k}+\boldsymbol{q})^{2}-\boldsymbol{k}^{2}-q^{2}}{2|\boldsymbol{k}||\boldsymbol{k}+\boldsymbol{q}|} \sum_{\lambda, \lambda^{\prime}= \pm} \lambda \lambda^{\prime} \mathcal{F}_{\lambda \lambda^{\prime}}(\boldsymbol{k}, \boldsymbol{k}+\boldsymbol{q}, \omega), \\
B_{a}(\boldsymbol{q}, \omega, \chi)=\frac{1}{\mathcal{V}} \sum_{\boldsymbol{k}} \frac{|\boldsymbol{k}|}{|\boldsymbol{k}+\boldsymbol{q}|} \sum_{\lambda, \lambda^{\prime}= \pm} \lambda \lambda^{\prime} \mathcal{F}_{\lambda \lambda^{\prime}}(\boldsymbol{k}, \boldsymbol{k}+\boldsymbol{q}, \omega), \\
q^{2} B_{b}(\boldsymbol{q}, \omega, \chi)=\frac{1}{\mathcal{V}} \sum_{\boldsymbol{k}} \frac{\left((\boldsymbol{k}+\boldsymbol{q})^{2}-\boldsymbol{k}^{2}-q^{2}\right)^{2}}{4|\boldsymbol{k}||\boldsymbol{k}+\boldsymbol{q}|} \sum_{\lambda, \lambda^{\prime}= \pm} \lambda \lambda^{\prime} \mathcal{F}_{\lambda \lambda^{\prime}}(\boldsymbol{k}, \boldsymbol{k}+\boldsymbol{q}, \omega), \\
C_{0}(\boldsymbol{q}, \omega, \chi)=\frac{1}{\mathcal{V}} \sum_{\boldsymbol{k}} \frac{1}{|\boldsymbol{k}+\boldsymbol{q}|} \sum_{\lambda, \lambda^{\prime}= \pm} \lambda^{\prime} \mathcal{F}_{\lambda \lambda^{\prime}}(\boldsymbol{k}, \boldsymbol{k}+\boldsymbol{q}, \omega), \\
q_{1}^{2}(\boldsymbol{q}, \omega, \chi)=\frac{1}{\mathcal{V}} \sum_{\boldsymbol{k}} \frac{(\boldsymbol{k}+\boldsymbol{q})^{2}-\boldsymbol{k}^{2}-q^{2}}{2|\boldsymbol{k}||\boldsymbol{k}+\boldsymbol{q}|} \sum_{\lambda, \lambda^{\prime}= \pm} \lambda^{\prime} \mathcal{F}_{\lambda \lambda^{\prime}}(\boldsymbol{k}, \boldsymbol{k}+\boldsymbol{q}, \omega), \\
q^{2} D_{1}(\boldsymbol{q}, \omega, \chi)=\frac{1}{\mathcal{V}} \sum_{\boldsymbol{k}} \frac{(\boldsymbol{k}+\boldsymbol{q})^{2}-\boldsymbol{k}^{2}-q^{2}}{2|\boldsymbol{k}||\boldsymbol{k}+\boldsymbol{q}|} \sum_{\lambda, \lambda^{\prime}= \pm} \lambda \mathcal{F}_{\lambda \lambda^{\prime}}(\boldsymbol{k}, \boldsymbol{k}+\boldsymbol{q}, \omega),
\end{gathered}
$$

where

$$
\mathcal{F}_{\lambda \lambda^{\prime}}(\boldsymbol{k}, \boldsymbol{k}+\boldsymbol{q}, \omega)=\frac{n_{F}\left[\varepsilon_{\lambda}(\boldsymbol{k})\right]-n_{F}\left[\varepsilon_{\lambda^{\prime}}(\boldsymbol{k}+\boldsymbol{q})\right]}{\omega+\varepsilon_{\lambda}(\boldsymbol{k})-\varepsilon_{\lambda^{\prime}}(\boldsymbol{k}+\boldsymbol{q})+i \eta},
$$

with $n_{F}(x)=1 /\left[\exp \left\{\beta_{T}\left(x-\mu_{\chi}\right)\right\}+1\right]$ being the Fermi distribution function and $\varepsilon_{\lambda}(\boldsymbol{k})=\lambda|\boldsymbol{k}|$.

\section{APPENDIX B: EXPRESSIONS OF SEVEN SCALAR INTEGRALS}

In this Appendix, we list the final expressions of the seven scalar integrals at zero temperature where the Fermi distribution function $n_{F}\left[\varepsilon_{\lambda}(\boldsymbol{k})\right]$ reduces to be the Heaviside step function $\Theta\left(\mu_{\chi}-\lambda|\boldsymbol{k}|\right)$. In order to express the analytical results in a compact form, we introduce the following auxiliary functions:

$$
\begin{gathered}
T(u)=\frac{1}{3} u^{3}-\omega^{2} u, \\
U(u)=\omega u^{2}-2 q^{2} u, \\
V(u)=\frac{1}{3} u^{3}-\omega u^{2}+\omega^{2} u, \\
W(u)=\frac{1}{3} \omega^{2} u^{3}-q^{2} \omega u^{2}+q^{4} u,
\end{gathered}
$$




$$
\begin{gathered}
X(u)=u^{2}-2 \omega u, \\
Y(u)=\frac{1}{3} \omega u^{3}-\frac{\left(q^{2}+\omega^{2}\right)}{2} u^{2}+q^{2} \omega u, \\
Z(u)=\frac{1}{3} \omega u^{3}-\frac{\left(q^{2}-\omega^{2}\right)}{2} u^{2}-q^{2} \omega u, \\
H(q, \omega)=\log \left|\frac{2 \mu_{\chi}+\omega-q}{\omega-q}\right| .
\end{gathered}
$$

After lengthy and complicated calculations, one finds the explicit expressions of the seven scalar integrals $A_{0}^{ \pm}, B_{1}^{ \pm}$, $B_{a}^{ \pm}, B_{b}^{ \pm}, C_{0}^{ \pm}, C_{1}^{ \pm}$, and $D_{1}^{ \pm}$, as follows.

\section{Expression of $A_{0}(q, \omega, \chi)$}

The scalar integral $A_{0}(\boldsymbol{q}, \omega, \chi)$ can de decomposed into the intrinsic and extrinsic part as follows:

$$
A_{0}=\frac{1}{\mathcal{V}} \sum_{\boldsymbol{k}} \sum_{\lambda= \pm} \sum_{\lambda^{\prime}= \pm} \frac{n_{F}\left[\varepsilon_{\lambda}(\boldsymbol{k})\right]-n_{F}\left[\varepsilon_{\lambda^{\prime}}(\boldsymbol{k}+\boldsymbol{q})\right]}{\omega+\varepsilon_{\lambda}(\boldsymbol{k})-\varepsilon_{\lambda^{\prime}}(\boldsymbol{k}+\boldsymbol{q})+i \eta}=A_{0}^{-}+A_{0}^{+},
$$

with the intrinsic part

$$
\begin{gathered}
\operatorname{Im} A_{0}^{-}=\frac{\left(q^{2}-3 \omega^{2}\right)}{48 \pi} \Theta(\omega-q), \\
\operatorname{Re} A_{0}^{-}=\frac{\left(q^{2}-6 \Lambda^{2}\right)}{24 \pi^{2}}+\frac{\left(q^{2}-3 \omega^{2}\right)}{48 \pi^{2}} \log \left|\frac{4 \Lambda^{2}}{q^{2}-\omega^{2}}\right|,
\end{gathered}
$$

and the extrinsic part

$$
\begin{aligned}
& \operatorname{Im} A_{0}^{+}= \frac{1}{32 \pi q}\left\{-\Theta(q-\omega) \sum_{\lambda= \pm} \lambda \Theta\left(\mu_{\chi}-\frac{q-\lambda \omega}{2}\right)\left[T\left(2 \mu_{\chi}+\lambda \omega\right)-T(q)\right]-\Theta(\omega-q)\right. \\
&\left.\times\left[\Theta\left(\mu_{\chi}-\frac{\omega+q}{2}\right)[T(q)-T(-q)]+\tilde{\Theta}\left(\frac{\omega+q}{2}-\mu_{\chi}\right) \Theta\left(\mu_{\chi}-\frac{\omega-q}{2}\right)\left[T\left(2 \mu_{\chi}-\omega\right)-T(-q)\right]\right]\right\}, \\
& \operatorname{Re} A_{0}^{+}=\frac{1}{32 \pi^{2} q}\left\{\sum_{\lambda= \pm} \sum_{\lambda^{\prime}= \pm} \lambda\left[T\left(2 \mu_{\chi}+\lambda^{\prime} \omega\right)-T(\lambda q)\right] H\left(\lambda q, \lambda^{\prime} \omega\right)-\frac{8 q \mu_{\chi}^{2}}{3}\right\} .
\end{aligned}
$$

\section{Expression of $B_{1}(q, \omega, \chi)$}

The scalar integral $B_{1}(\boldsymbol{q}, \omega, \chi)$ is given as

$$
q^{2} B_{1}=\frac{1}{\mathcal{V}} \sum_{\boldsymbol{k}} \sum_{\lambda= \pm} \sum_{\lambda^{\prime}= \pm} \lambda \lambda^{\prime} \frac{(\boldsymbol{k}+\boldsymbol{q})^{2}-\boldsymbol{k}^{2}-q^{2}}{2|\boldsymbol{k}||\boldsymbol{k}+\boldsymbol{q}|} \frac{n_{F}\left[\varepsilon_{\lambda}(\boldsymbol{k})\right]-n_{F}\left[\varepsilon_{\lambda^{\prime}}(\boldsymbol{k}+\boldsymbol{q})\right]}{\omega+\varepsilon_{\lambda}(\boldsymbol{k})-\varepsilon_{\lambda^{\prime}}(\boldsymbol{k}+\boldsymbol{q})+i \eta}=q^{2} B_{1}^{-}+q^{2} B_{1}^{+},
$$

with the intrinsic part

$$
\begin{gathered}
q^{2} \operatorname{Im} B_{1}^{-}=-\frac{q^{2}}{8 \pi} \Theta(\omega-q), \\
q^{2} \operatorname{Re} B_{1}^{-}=\frac{q^{2}}{12 \pi^{2}}-\frac{q^{2}}{8 \pi^{2}} \log \left|\frac{4 \Lambda^{2}}{q^{2}-\omega^{2}}\right|,
\end{gathered}
$$

and the extrinsic part

$$
\begin{gathered}
q^{2} \operatorname{Im} B_{1}^{+}=\frac{1}{32 \pi q}\left\{-\Theta(q-\omega) \sum_{\lambda= \pm} \lambda \Theta\left(\mu_{\chi}-\frac{q-\lambda \omega}{2}\right)\left[U\left(2 \mu_{\chi}+\lambda \omega\right)-U(q)\right]-\Theta(\omega-q)\right. \\
\left.\times\left[\Theta\left(\mu_{\chi}-\frac{\omega+q}{2}\right)[U(q)-U(-q)]+\Theta\left(\frac{\omega+q}{2}-\mu_{\chi}\right) \Theta\left(\mu_{\chi}-\frac{\omega-q}{2}\right)\left[U\left(2 \mu_{\chi}-\omega\right)-U(-q)\right]\right]\right\}, \\
q^{2} \operatorname{Re} B_{1}^{+}=\frac{1}{32 \pi^{2} q}\left\{\sum_{\lambda= \pm} \sum_{\lambda^{\prime}= \pm} \lambda\left[U\left(2 \mu_{\chi}+\lambda^{\prime} \omega\right)-U(\lambda q)\right] H\left(\lambda q, \lambda^{\prime} \omega\right)-8 q \omega \mu_{\chi}\right\} .
\end{gathered}
$$




\section{Expression of $B_{a}(q, \omega, \chi)$}

The scalar integral $B_{a}(\boldsymbol{q}, \omega, \chi)$ is given as

$$
B_{a}=\frac{1}{\mathcal{V}} \sum_{\boldsymbol{k}} \sum_{\lambda= \pm} \sum_{\lambda^{\prime}= \pm} \lambda \lambda^{\prime} \frac{|\boldsymbol{k}|}{|\boldsymbol{k}+\boldsymbol{q}|} \frac{n_{F}\left[\varepsilon_{\lambda}(\boldsymbol{k})\right]-n_{F}\left[\varepsilon_{\lambda^{\prime}}(\boldsymbol{k}+\boldsymbol{q})\right]}{\omega+\varepsilon_{\lambda}(\boldsymbol{k})-\varepsilon_{\lambda^{\prime}}(\boldsymbol{k}+\boldsymbol{q})+i \eta}=q^{2} B_{a}^{-}+q^{2} B_{a}^{+},
$$

with the intrinsic part

$$
\begin{gathered}
\operatorname{Im} B_{a}^{-}=\frac{\left(q^{2}+3 \omega^{2}\right)}{48 \pi} \Theta(\omega-q), \\
\operatorname{Re} B_{a}^{-}=\frac{\left(2 \Lambda^{2}-q^{2}\right)}{8 \pi^{2}}+\frac{\left(q^{2}+3 \omega^{2}\right)}{48 \pi^{2}} \log \left|\frac{4 \Lambda^{2}}{q^{2}-\omega^{2}}\right|,
\end{gathered}
$$

and the extrinsic part

$$
\begin{aligned}
\operatorname{Im} B_{a}^{+}= & \frac{1}{32 \pi q}\left\{-\Theta(q-\omega) \sum_{\lambda= \pm} \lambda \Theta\left(\mu_{\chi}-\frac{q-\lambda \omega}{2}\right)\left[V\left(2 \mu_{\chi}+\lambda \omega\right)-V(q)\right]-\Theta(\omega-q)\right. \\
& \left.\times\left[\Theta\left(\mu_{\chi}-\frac{\omega+q}{2}\right)[V(q)-V(-q)]+\tilde{\Theta}\left(\frac{\omega+q}{2}-\mu_{\chi}\right) \Theta\left(\mu_{\chi}-\frac{\omega-q}{2}\right)\left[V\left(2 \mu_{\chi}-\omega\right)-V(-q)\right]\right]\right\} \\
& \operatorname{Re} B_{a}^{+}=\frac{1}{32 \pi^{2} q}\left\{\sum_{\lambda= \pm} \sum_{\lambda^{\prime}= \pm} \lambda\left[V\left(2 \mu_{\chi}+\lambda^{\prime} \omega\right)-V(\lambda q)\right] H\left(\lambda q, \lambda^{\prime} \omega\right)-\frac{8}{3} q \mu_{\chi}\left(7 \mu_{\chi}-3 \omega\right)\right\} .
\end{aligned}
$$

\section{Expression of $B_{b}(q, \omega, \chi)$}

The scalar integral $B_{b}(\boldsymbol{q}, \omega, \chi)$ is given as

$$
q^{2} B_{b}=\frac{1}{\mathcal{V}} \sum_{\boldsymbol{k}} \sum_{\lambda= \pm} \sum_{\lambda^{\prime}= \pm} \lambda \lambda^{\prime} \frac{\left[(\boldsymbol{k}+\boldsymbol{q})^{2}-\boldsymbol{k}^{2}-q^{2}\right]^{2}}{4|\boldsymbol{k}||\boldsymbol{k}+\boldsymbol{q}|} \frac{n_{F}\left[\varepsilon_{\lambda}(\boldsymbol{k})\right]-n_{F}\left[\varepsilon_{\lambda^{\prime}}(\boldsymbol{k}+\boldsymbol{q})\right]}{\omega+\varepsilon_{\lambda}(\boldsymbol{k})-\varepsilon_{\lambda^{\prime}}(\boldsymbol{k}+\boldsymbol{q})+i \eta}=q^{2} B_{b}^{-}+q^{2} B_{b}^{+},
$$

with the intrinsic part

$$
\begin{gathered}
q^{2} \operatorname{Im} B_{b}^{-}=\frac{q^{2}\left(3 q^{2}+\omega^{2}\right)}{48 \pi} \Theta(\omega-q), \\
q^{2} \operatorname{Re} B_{b}^{-}=\frac{q^{2}\left(10 \Lambda^{2}-11 q^{2}\right)}{120 \pi^{2}}+\frac{q^{2}\left(3 q^{2}+\omega^{2}\right)}{48 \pi^{2}} \log \left|\frac{4 \Lambda^{2}}{q^{2}-\omega^{2}}\right|,
\end{gathered}
$$

and the extrinsic part

$$
\begin{aligned}
q^{2} \operatorname{Im} B_{b}^{+}= & \frac{1}{32 \pi q}\left\{-\Theta(q-\omega) \sum_{\lambda= \pm} \lambda \Theta\left(\mu_{\chi}-\frac{q-\lambda \omega}{2}\right)\left[W\left(2 \mu_{\chi}+\lambda \omega\right)-W(q)\right]-\Theta(\omega-q)\right. \\
& \left.\times\left[\Theta\left(\mu_{\chi}-\frac{\omega+q}{2}\right)[W(q)-W(-q)]+\tilde{\Theta}\left(\frac{\omega+q}{2}-\mu_{\chi}\right) \Theta\left(\mu_{\chi}-\frac{\omega-q}{2}\right)\left[W\left(2 \mu_{\chi}-\omega\right)-W(-q)\right]\right]\right\} \\
& q^{2} \operatorname{Re} B_{b}^{+}=\frac{1}{32 \pi^{2} q}\left\{\sum_{\lambda= \pm} \sum_{\lambda^{\prime}= \pm} \lambda\left[W\left(2 \mu_{\chi}+\lambda^{\prime} \omega\right) W(\lambda q)\right] H\left(\lambda q, \lambda^{\prime} \omega\right)-\frac{32}{3} q \omega^{2} \mu_{\chi}^{2}-8 q^{3} \mu_{\chi}\left(\mu_{\chi}-\omega\right)\right\}
\end{aligned}
$$

The scalar integral $C_{0}(\boldsymbol{q}, \omega, \chi)$ is given as

\section{Expression of $C_{0}(q, \omega, \chi)$}

$$
C_{0}=\frac{1}{\mathcal{V}} \sum_{\boldsymbol{k}} \sum_{\lambda= \pm} \sum_{\lambda^{\prime}= \pm} \lambda^{\prime} \frac{1}{|\boldsymbol{k}+\boldsymbol{q}|} \frac{n_{F}\left[\varepsilon_{\lambda}(\boldsymbol{k})\right]-n_{F}\left[\varepsilon_{\lambda^{\prime}}(\boldsymbol{k}+\boldsymbol{q})\right]}{\omega+\varepsilon_{\lambda}(\boldsymbol{k})-\varepsilon_{\lambda^{\prime}}(\boldsymbol{k}+\boldsymbol{q})+i \eta}=C_{0}^{-}+C_{0}^{+},
$$

with the intrinsic part

$$
\begin{gathered}
\operatorname{Im} C_{0}^{-}=-\frac{\omega}{8 \pi} \Theta(\omega-q), \\
\operatorname{Re} C_{0}^{-}=-\frac{\omega}{8 \pi^{2}} \log \left|\frac{4 \Lambda^{2}}{q^{2}-\omega^{2}}\right|,
\end{gathered}
$$


and the extrinsic part

$$
\begin{aligned}
\operatorname{Im} C_{0}^{+}= & \frac{1}{32 \pi q}\left\{-\Theta(q-\omega) \sum_{\lambda= \pm} \lambda \Theta\left(\mu_{\chi}-\frac{q-\lambda \omega}{2}\right)\left[X\left(2 \mu_{\chi}+\lambda \omega\right)-X(q)\right]-\Theta(\omega-q)\right. \\
& \times\left[\Theta\left(\mu_{\chi}-\frac{\omega+q}{2}\right)[X(q)-X(-q)]+\tilde{\Theta}\left(\frac{\omega+q}{2}-\mu_{\chi}\right) \Theta\left(\mu_{\chi}-\frac{\omega-q}{2}\right)\left[X\left(2 \mu_{\chi}-\omega\right)-X(-q)\right]\right], \\
& \operatorname{Re} C_{0}^{+}=\frac{1}{32 \pi^{2} q}\left\{\sum_{\lambda= \pm} \sum_{\lambda^{\prime}= \pm} \lambda\left[X\left(2 \mu_{\chi}+\lambda^{\prime} \omega\right)-X(\lambda q)\right] H\left(\lambda q, \lambda^{\prime} \omega\right)-8 q \mu_{\chi}\right\} .
\end{aligned}
$$

\section{Expression of $C_{1}(q, \omega, \chi)$}

The scalar integral $C_{1}(\boldsymbol{q}, \omega, \chi)$ is given as

$$
q^{2} C_{1}=\frac{1}{\mathcal{V}} \sum_{\boldsymbol{k}} \sum_{\lambda= \pm} \sum_{\lambda^{\prime}= \pm} \lambda^{\prime} \frac{(\boldsymbol{k}+\boldsymbol{q})^{2}-\boldsymbol{k}^{2}-q^{2}}{2|\boldsymbol{k}+\boldsymbol{q}|} \frac{n_{F}\left[\varepsilon_{\lambda}(\boldsymbol{k})\right]-n_{F}\left[\varepsilon_{\lambda^{\prime}}(\boldsymbol{k}+\boldsymbol{q})\right]}{\omega+\varepsilon_{\lambda}(\boldsymbol{k})-\varepsilon_{\lambda^{\prime}}(\boldsymbol{k}+\boldsymbol{q})+i \eta}=q^{2} C_{1}^{-}+q^{2} C_{1}^{+},
$$

with the intrinsic part

$$
\begin{gathered}
q^{2} \operatorname{Im} C_{1}^{-}=\frac{q^{2} \omega}{12 \pi} \Theta(\omega-q), \\
q^{2} \operatorname{Re} C_{1}^{-}=-\frac{q^{2} \omega}{24 \pi^{2}}+\frac{q^{2} \omega}{12 \pi^{2}} \log \left|\frac{4 \Lambda^{2}}{q^{2}-\omega^{2}}\right|,
\end{gathered}
$$

and the extrinsic part

$$
\begin{aligned}
q^{2} \operatorname{Im} C_{1}^{+}= & \frac{1}{32 \pi q}\left\{-\Theta(q-\omega) \sum_{\lambda= \pm} \lambda \Theta\left(\mu_{\chi}-\frac{q-\lambda \omega}{2}\right)\left[Y\left(2 \mu_{\chi}+\lambda \omega\right)-Y(q)\right]-\Theta(\omega-q)\right. \\
& \left.\times\left[\Theta\left(\mu_{\chi}-\frac{\omega+q}{2}\right)[Y(q)-Y(-q)]+\tilde{\Theta}\left(\frac{\omega+q}{2}-\mu_{\chi}\right) \Theta\left(\mu_{\chi}-\frac{\omega-q}{2}\right)\left[Y\left(2 \mu_{\chi}-\omega\right)-Y(-q)\right]\right]\right\}, \\
& q^{2} \operatorname{Re} C_{1}^{+}=\frac{1}{32 \pi^{2} q}\left\{\sum_{\lambda= \pm} \sum_{\lambda^{\prime}= \pm} \lambda\left[Y\left(2 \mu_{\chi}+\lambda^{\prime} \omega\right)-Y(\lambda q)\right] H\left(\lambda q, \lambda^{\prime} \omega\right)-\frac{32}{3} q \omega \mu_{\chi}^{2}+4 q\left(q^{2}+\omega^{2}\right) \mu_{\chi}\right\} .
\end{aligned}
$$

\section{Expression of $D_{1}(q, \omega, \chi)$}

The scalar integral $D_{1}(\boldsymbol{q}, \omega, \chi)$ is given as

$$
q^{2} D_{1}=\frac{1}{\mathcal{V}} \sum_{\boldsymbol{k}} \sum_{\lambda= \pm} \sum_{\lambda^{\prime}= \pm} \lambda \frac{(\boldsymbol{k}+\boldsymbol{q})^{2}-\boldsymbol{k}^{2}-q^{2}}{2|\boldsymbol{k}|} \frac{n_{F}\left[\varepsilon_{\lambda}(\boldsymbol{k})\right]-n_{F}\left[\varepsilon_{\lambda^{\prime}}(\boldsymbol{k}+\boldsymbol{q})\right]}{\omega+\varepsilon_{\lambda}(\boldsymbol{k})-\varepsilon_{\lambda^{\prime}}(\boldsymbol{k}+\boldsymbol{q})+i \eta}=q^{2} D_{1}^{-}+q^{2} D_{1}^{+},
$$

with the intrinsic part

$$
\begin{gathered}
q^{2} \operatorname{Im} D_{1}^{-}=-\frac{q^{2} \omega}{24 \pi} \Theta(\omega-q), \\
q^{2} \operatorname{Re} D_{1}^{-}=\frac{q^{2} \omega}{24 \pi^{2}}-\frac{q^{2} \omega}{24 \pi^{2}} \log \left|\frac{4 \Lambda^{2}}{q^{2}-\omega^{2}}\right|,
\end{gathered}
$$

and the extrinsic part

$$
\begin{aligned}
q^{2} \operatorname{Im} D_{1}^{+}= & \frac{1}{32 \pi q}\left\{-\Theta(q-\omega) \sum_{\lambda= \pm} \lambda \Theta\left(\mu_{\chi}-\frac{q-\lambda \omega}{2}\right)\left[Z\left(2 \mu_{\chi}+\lambda \omega\right)-Z(q)\right]-\Theta(\omega-q)\right. \\
& \left.\times\left[\Theta\left(\mu_{\chi}-\frac{\omega+q}{2}\right)[Z(q)-Z(-q)]+\tilde{\Theta}\left(\frac{\omega+q}{2}-\mu_{\chi}\right) \Theta\left(\mu_{\chi}-\frac{\omega-q}{2}\right)\left[Z\left(2 \mu_{\chi}-\omega\right)-Z(-q)\right]\right]\right\}, \\
& q^{2} \operatorname{Re} D_{1}^{+}=\frac{1}{32 \pi^{2} q}\left\{\sum_{\lambda= \pm} \sum_{\lambda^{\prime}= \pm} \lambda\left[Z\left(2 \mu_{\chi}+\lambda^{\prime} \omega\right)-Z(\lambda q)\right] H\left(\lambda q, \lambda^{\prime} \omega\right)-\frac{32}{3} q \omega \mu_{\chi}^{2}+4 q\left(q^{2}-\omega^{2}\right) \mu_{\chi}\right\} .
\end{aligned}
$$




\section{APPENDIX C: EVALUATION OF $A_{0}(q, \omega, \chi)$}

In this Appendix, we take $A_{0}(\boldsymbol{q}, \omega, \chi)$ as an example to outline the main procedure and tricks to evaluate the seven scalar integrals at zero temperature.

\section{Intrinsic case}

For the intrinsic case $\mu_{\chi}=0$, at zero temperature the Fermi distribution function $n_{F}[x]=\Theta\left(\mu_{\chi}-x\right)$, leading to $A_{0}^{+}=0$ and $A_{0}^{-} \neq 0$.

$$
A_{0}^{-}=\frac{1}{\mathcal{V}} \sum_{\boldsymbol{k}}\left\{\sum_{\lambda^{\prime}= \pm} \frac{n_{F}\left[\varepsilon_{-}(\boldsymbol{k})\right]}{\omega+\varepsilon_{-}(\boldsymbol{k})-\varepsilon_{\lambda^{\prime}}(\boldsymbol{k}+\boldsymbol{q})+i \eta}-\sum_{\lambda= \pm} \frac{n_{F}\left[\varepsilon_{-}(\boldsymbol{k}+\boldsymbol{q})\right]}{\omega+\varepsilon_{\lambda}(\boldsymbol{k})-\varepsilon_{-}(\boldsymbol{k}+\boldsymbol{q})+i \eta}\right\}=A_{0 a}^{-}+A_{0 b}^{-} \text {. }
$$

It is noted that the other two terms in the curly brackets cancel each other when the line in $A_{0}^{-}$vanishes. The left two terms can be evaluated as

$$
\begin{aligned}
A_{0 a}^{-} & =\operatorname{Re} A_{0 a}^{-}+i \operatorname{Im} A_{0 a}^{-}=\frac{1}{\mathcal{V}} \sum_{\boldsymbol{k}} \frac{n_{F}\left[\varepsilon_{-}(\boldsymbol{k})\right]}{\omega+\varepsilon_{-}(\boldsymbol{k})-\varepsilon_{+}(\boldsymbol{k}+\boldsymbol{q})+i \eta}=\frac{1}{\mathcal{V}} \sum_{\boldsymbol{k}} \frac{1}{\omega+\varepsilon_{-}(\boldsymbol{k})-\varepsilon_{+}(\boldsymbol{k}+\boldsymbol{q})+i \eta} \\
& =\frac{1}{4 \pi^{2} q} \int_{0}^{\Lambda} k d k \int_{|k-q|}^{k+q} y d y \frac{1}{\omega-k-y+i \eta}=\frac{1}{4 \pi^{2} q} \int_{0}^{\Lambda} k d k \int_{|k-q|}^{k+q} y d y\left\{\mathcal{P} \frac{1}{\omega-k-y}-i \pi \delta(\omega-k-y)\right\}
\end{aligned}
$$

similarly

$$
\begin{aligned}
A_{0 b}^{-} & =\operatorname{Re} A_{0 b}^{-}+i \operatorname{Im} A_{0 b}^{-}=\frac{1}{\mathcal{V}} \sum_{\boldsymbol{k}} \frac{-n_{F}\left[\varepsilon_{-}(\boldsymbol{k}+\boldsymbol{q})\right]}{\omega+\varepsilon_{+}(\boldsymbol{k})-\varepsilon_{-}(\boldsymbol{k}+\boldsymbol{q})+i \eta}=\frac{1}{\mathcal{V}} \sum_{\boldsymbol{k}} \frac{-1}{\omega+\varepsilon_{+}(\boldsymbol{k})-\varepsilon_{-}(\boldsymbol{k}+\boldsymbol{q})+i \eta} \\
& =\frac{-1}{4 \pi^{2} q} \int_{0}^{\Lambda} k d k \int_{|k-q|}^{k+q} y d y \frac{1}{\omega+k+y+i \eta}=\frac{-1}{4 \pi^{2} q} \int_{0}^{\Lambda} k d k \int_{|k-q|}^{k+q} y d y\left\{\mathcal{P} \frac{1}{\omega+k+y}-i \pi \delta(\omega+k+y)\right\} .
\end{aligned}
$$

Integrating over $y$ and $k$ leads to $A_{0}^{-}$for the intrinsic case in Eqs. (B10) and (B11).

\section{Extrinsic case}

Let us calculate the extrinsic case $\mu_{\chi}>0$ as follows:

$$
A_{0}^{+}=\frac{1}{\mathcal{V}} \sum_{\boldsymbol{k}}\left\{\sum_{\lambda^{\prime}= \pm} \frac{n_{F}\left[\varepsilon_{+}(\boldsymbol{k})\right]}{\omega+\varepsilon_{+}(\boldsymbol{k})-\varepsilon_{\lambda^{\prime}}(\boldsymbol{k}+\boldsymbol{q})+i \eta}-\sum_{\lambda= \pm} \frac{n_{F}\left[\varepsilon_{+}(\boldsymbol{k}+\boldsymbol{q})\right]}{\omega+\varepsilon_{\lambda}(\boldsymbol{k})-\varepsilon_{+}(\boldsymbol{k}+\boldsymbol{q})+i \eta}\right\}=A_{0 a}^{+}+A_{0 b}^{+},
$$

where $A_{0 a}^{+}$and $A_{0 b}^{+}$are given as

$$
\begin{aligned}
A_{0 a}^{+} & =\frac{1}{\mathcal{V}} \sum_{\boldsymbol{k}} n_{F}\left[\varepsilon_{+}(\boldsymbol{k})\right]\left\{\frac{1}{\omega+\varepsilon_{+}(\boldsymbol{k})-\varepsilon_{-}(\boldsymbol{k}+\boldsymbol{q})+i \eta}+\frac{1}{\omega+\varepsilon_{+}(\boldsymbol{k})-\varepsilon_{+}(\boldsymbol{k}+\boldsymbol{q})+i \eta}\right\} \\
& =\frac{1}{4 \pi^{2} q} \int_{0}^{\Lambda} k d k \int_{|k-q|}^{k+q} y d y \Theta\left(\mu_{\chi}-k\right)\left\{\frac{1}{\omega+k+y+i \eta}+\frac{1}{\omega+k-y+i \eta}\right\} \\
& =\frac{1}{4 \pi^{2} q} \int_{0}^{\mu_{x}} k d k \int_{|k-q|}^{k+q} y d y\left\{\mathcal{P} \frac{2(\omega+k)}{(\omega+k)^{2}-y^{2}}-2 i \pi|\omega+k| \delta\left[(\omega+k)^{2}-y^{2}\right]\right\},
\end{aligned}
$$

and

$$
\begin{aligned}
A_{0 b}^{+} & =\frac{1}{\mathcal{V}} \sum_{\boldsymbol{k}} n_{F}\left[\varepsilon_{+}(\boldsymbol{k})\right]\left\{\frac{1}{\omega-\varepsilon_{+}(\boldsymbol{k})+\varepsilon_{-}(\boldsymbol{k}+\boldsymbol{q})+i \eta}+\frac{1}{\omega-\varepsilon_{+}(\boldsymbol{k})+\varepsilon_{+}(\boldsymbol{k}+\boldsymbol{q})+i \eta}\right\} \\
& =\frac{-1}{4 \pi^{2} q} \int_{0}^{\Lambda} k d k \int_{|k-q|}^{k+q} y d y \Theta\left(\mu_{\chi}-k\right)\left\{\frac{1}{\omega-k-y+i \eta}+\frac{1}{\omega-k+y+i \eta}\right\} \\
& =\frac{-1}{4 \pi^{2} q} \int_{0}^{\mu_{x}} k d k \int_{|k-q|}^{k+q} y d y\left\{\mathcal{P}\left[\frac{2(\omega-k)}{(\omega-k)^{2}-y^{2}}\right]-2 i \pi|\omega-k| \delta\left[(\omega-k)^{2}-y^{2}\right]\right\} .
\end{aligned}
$$

After integrating over $y$ and $k$, we obtain $A_{0}^{+}$for the extrinsic case in Eqs. (B12) and (B13). 


\section{APPENDIX D: CAUSALITY RELATIONS}

Let us rewrite the chirality-dependent correlation functions $\Pi^{00}(\boldsymbol{q}, \omega, \chi), \Pi^{\alpha \beta}(\boldsymbol{q}, \omega, \chi)$, and $\Pi^{0 \alpha / \alpha 0}(\boldsymbol{q}, \omega, \chi)$ as

$$
\begin{gathered}
\Pi^{00}(\boldsymbol{q}, \omega, \chi) \equiv \frac{1}{2}\left[A_{0}(\boldsymbol{q}, \omega, \chi)+B_{a}(\boldsymbol{q}, \omega, \chi)+q^{2} B_{1}(\boldsymbol{q}, \omega, \chi)\right], \\
\Pi^{\alpha \beta}(\boldsymbol{q}, \omega, \chi) \equiv F_{T}(\boldsymbol{q}, \omega, \chi)\left(\delta^{\alpha \beta}-\frac{q^{\alpha} q^{\beta}}{q^{2}}\right)+F_{L}(\boldsymbol{q}, \omega, \chi) \frac{q^{\alpha} q^{\beta}}{q^{2}}+i \chi F_{A}(\boldsymbol{q}, \omega, \chi) \varepsilon^{\alpha \beta \tau} q^{\tau}, \\
\Pi^{\alpha 0}(\boldsymbol{q}, \omega, \chi)=\Pi^{0 \alpha}(\boldsymbol{q}, \omega, \chi) \equiv F_{I}(\boldsymbol{q}, \omega, \chi) q^{\alpha}
\end{gathered}
$$

where the four auxiliary functions read

$$
\begin{gathered}
F_{T}(\boldsymbol{q}, \omega, \chi)=\frac{1}{2}\left[A_{0}(\boldsymbol{q}, \omega, \chi)-B_{b}(\boldsymbol{q}, \omega, \chi)-q^{2} B_{1}(\boldsymbol{q}, \omega, \chi)\right], \\
F_{L}(\boldsymbol{q}, \omega, \chi)=\frac{1}{2}\left[A_{0}(\boldsymbol{q}, \omega, \chi)-B_{a}(\boldsymbol{q}, \omega, \chi)+2 B_{b}(\boldsymbol{q}, \omega, \chi)+q^{2} B_{1}(\boldsymbol{q}, \omega, \chi)\right], \\
F_{A}(\boldsymbol{q}, \omega, \chi)=-\frac{1}{2}\left[C_{1}(\boldsymbol{q}, \omega, \chi)+C_{0}(\boldsymbol{q}, \omega, \chi)-D_{1}(\boldsymbol{q}, \omega, \chi)\right], \\
F_{I}(\boldsymbol{q}, \omega, \chi)=\frac{1}{2}\left[C_{1}(\boldsymbol{q}, \omega, \chi)+C_{0}(\boldsymbol{q}, \omega, \chi)+D_{1}(\boldsymbol{q}, \omega, \chi)\right] .
\end{gathered}
$$

Next we take $\Pi^{0 \alpha / \alpha 0}(\boldsymbol{q}, \omega, \chi)$ as an example to derive the causality relation of each chirality-dependent correlation function. Let us first take the Hermitian conjugate of $\Pi^{0 \alpha}(\boldsymbol{q}, \omega, \chi)$ as

$$
\left[\Pi^{0 \alpha}(\boldsymbol{q}, \omega, \chi)\right]^{\dagger}=\left[\Pi^{\alpha 0}(\boldsymbol{q}, \omega, \chi)\right]^{*}=\left[\Pi^{0 \alpha}(\boldsymbol{q}, \omega, \chi)\right]^{*}=\left[F_{I}(\boldsymbol{q}, \omega, \chi)\right]^{*} q^{\alpha},
$$

where the complex conjugate of $F_{I}(\boldsymbol{q}, \omega, \chi)$ reads

$$
\left[F_{I}(\boldsymbol{q}, \omega, \chi)\right]^{*}=\frac{1}{2 \mathcal{V}} \sum_{\boldsymbol{k}} \sum_{\lambda, \lambda^{\prime}= \pm}\left(\lambda^{\prime} \frac{(\boldsymbol{k}+\boldsymbol{q})^{2}-\boldsymbol{k}^{2}+q^{2}}{2 q^{2}|\boldsymbol{k}+\boldsymbol{q}|}+\lambda \frac{(\boldsymbol{k}+\boldsymbol{q})^{2}-\boldsymbol{k}^{2}-q^{2}}{2 q^{2}|\boldsymbol{k}|}\right) \frac{n_{F}\left[\varepsilon_{\lambda}(\boldsymbol{k})\right]-n_{F}\left[\varepsilon_{\lambda^{\prime}}(\boldsymbol{k}+\boldsymbol{q})\right]}{\omega+\varepsilon_{\lambda}(\boldsymbol{k})-\varepsilon_{\lambda^{\prime}}(\boldsymbol{k}+\boldsymbol{q})-i \eta} .
$$

Relabeling $(\boldsymbol{k}, \lambda) \leftrightarrow\left(-\boldsymbol{k}-\boldsymbol{q}, \lambda^{\prime}\right)$ leads to

$$
\begin{aligned}
{\left[F_{I}(\boldsymbol{q}, \omega, \chi)\right]^{*} } & =-\frac{1}{2 \mathcal{V}} \sum_{\boldsymbol{k}} \sum_{\lambda, \lambda^{\prime}= \pm}\left(\lambda^{\prime} \frac{(\boldsymbol{k}+\boldsymbol{q})^{2}-\boldsymbol{k}^{2}+q^{2}}{2 q^{2}|\boldsymbol{k}+\boldsymbol{q}|}+\lambda \frac{(\boldsymbol{k}+\boldsymbol{q})^{2}-\boldsymbol{k}^{2}-q^{2}}{2 q^{2}|\boldsymbol{k}|}\right) \frac{n_{F}\left[\varepsilon_{\lambda}(\boldsymbol{k})\right]-n_{F}\left[\varepsilon_{\lambda^{\prime}}(\boldsymbol{k}+\boldsymbol{q})\right]}{-\omega+\varepsilon_{\lambda}(\boldsymbol{k})-\varepsilon_{\lambda^{\prime}}(\boldsymbol{k}+\boldsymbol{q})+i \eta} \\
& =-F_{I}(\boldsymbol{q},-\omega, \chi),
\end{aligned}
$$

which implies

$$
\left[\Pi^{0 \alpha}(\boldsymbol{q}, \omega, \chi)\right]^{\dagger}=\left[\Pi^{\alpha 0}(\boldsymbol{q}, \omega, \chi)\right]^{*}=-F_{I}(\boldsymbol{q},-\omega, \chi) q^{\alpha}=-\Pi^{0 \alpha}(\boldsymbol{q},-\omega, \chi) .
$$

Other correlation functions can be calculated in a similar way. Finally, we have

$$
\begin{gathered}
\operatorname{Re} \Pi^{00}(\boldsymbol{q},-\omega, \chi)=\operatorname{Re} \Pi^{00}(\boldsymbol{q}, \omega, \chi), \\
\operatorname{Im} \Pi^{00}(\boldsymbol{q},-\omega, \chi)=-\operatorname{Im} \Pi^{00}(\boldsymbol{q}, \omega, \chi), \\
\operatorname{Re} F_{X}(\boldsymbol{q},-\omega, \chi)=\operatorname{Re} F_{X}(\boldsymbol{q}, \omega, \chi), \\
\operatorname{Im} F_{X}(\boldsymbol{q},-\omega, \chi)=-\operatorname{Im} F_{X}(\boldsymbol{q}, \omega, \chi), \\
\operatorname{Re} F_{I}(\boldsymbol{q},-\omega, \chi)=-\operatorname{Re} F_{I}(\boldsymbol{q}, \omega, \chi), \\
\operatorname{Im} F_{I}(\boldsymbol{q},-\omega, \chi)=\operatorname{Im} F_{I}(\boldsymbol{q}, \omega, \chi),
\end{gathered}
$$

which helps us to obtain the negative-frequency part of $\Pi^{\mu v}(\boldsymbol{q}, \omega, \chi)$ by taking the Hermitian conjugate of that with the positive frequency. Here $X=T, L, A$. Thus, we only need to consider the positive-frequency part $\omega>0$.

\section{APPENDIX E: KRAMERS-KRONIG RELATION WITH $n$ th-ORDER SUBTRACTION}

In this Appendix, we give a proof of the dispersion relation with $n$ th-order subtraction. For an analytic function $f(\xi)$ in the upper half plane, if $f(\xi)$ does not diverge more than $\xi^{n-1}$ as $\xi \rightarrow \infty$, we construct a Cauchy integral

$$
\mathcal{J}_{n-1}=\lim _{\eta \rightarrow 0^{+}} \frac{1}{2 \pi i} \oint_{C} d \xi \frac{f(\xi)}{[\xi-(\omega+i \eta)]} \prod_{m=1}^{n} \frac{1}{\left[\xi-\left(\omega_{m}+i \eta\right)\right]}
$$


which has $n+1$ poles at $\xi=\omega+i \eta, \xi=\omega_{m}+i \eta$ with $\omega \neq \omega_{m} \neq \omega_{l}, m, l=1,2,3, \ldots, n$, and $m \neq l$. The auxiliary function $\mathcal{J}_{n-1}$ can be calculated in two ways. The first one is

$$
\begin{aligned}
\mathcal{J}_{n-1} & =\lim _{\eta \rightarrow 0^{+}} \frac{1}{2 \pi i} \oint_{C} d \xi \frac{f(\xi)}{(\xi-\omega-i \eta)} \prod_{m=1}^{n} \frac{1}{\left[\xi-\left(\omega_{m}+i \eta\right)\right]} \\
& =\lim _{\eta \rightarrow 0^{+}}\left[\frac{(\xi-\omega-i \eta) f(\xi)}{(\xi-\omega-i \eta)} \prod_{m=1}^{n} \frac{1}{\left[\xi-\left(\omega_{m}+i \eta\right)\right]}\right]_{\xi=\omega+i \eta}+\sum_{l=1}^{n} \lim _{\eta \rightarrow 0^{+}}\left[\frac{\left(\xi-\omega_{l}-i \eta\right) f(\xi)}{(\xi-\omega-i \eta)} \prod_{m=1}^{n} \frac{1}{\left[\xi-\left(\omega_{m}+i \eta\right)\right]}\right]_{\xi=\omega_{l}+i \eta} \\
& =f(\omega) \prod_{m=1}^{n} \frac{1}{\left(\omega-\omega_{m}\right)}+\sum_{l=1}^{n} \frac{f\left(\omega_{l}\right)}{\left(\omega_{l}-\omega\right)} \prod_{m=1, m \neq l}^{n} \frac{1}{\left(\omega_{l}-\omega_{m}\right)}
\end{aligned}
$$

The second one is

$$
\begin{aligned}
\mathcal{J}_{n-1} & =\lim _{\eta \rightarrow 0^{+}} \frac{1}{2 \pi i} \oint_{C} d \xi \frac{f(\xi)}{[\xi-(\omega+i \eta)]} \prod_{m=1}^{n} \frac{1}{\left[\xi-\left(\omega_{m}+i \eta\right)\right]}=\lim _{\eta \rightarrow 0^{+}} \frac{1}{2 \pi i}\left(\int_{-\infty}^{+\infty} d \xi \frac{f(\xi)}{g(\xi)-i \eta g^{\prime}(\xi)}+i \pi \mathcal{C}_{\infty}\right) \\
& =\frac{1}{2 \pi i}\left(\mathcal{P} \int_{-\infty}^{+\infty} d \xi \frac{f(\xi)}{g(\xi)}+i \pi \int_{-\infty}^{+\infty} d \xi f(\xi) \operatorname{sgn}\left[g^{\prime}(\xi)\right] \delta[g(\xi)]+i \pi \mathcal{C}_{\infty}\right) \\
& =\frac{1}{2 \pi i} \mathcal{P} \int_{-\infty}^{+\infty} d \xi \frac{f(\xi)}{g(\xi)}+\frac{1}{2} \int_{-\infty}^{+\infty} d \xi f(\xi) \operatorname{sgn}\left[g^{\prime}(\xi)\right] \sum_{j=1}^{n+1} \frac{\delta\left(\xi-\xi_{0, j}\right)}{\left|g^{\prime}(\xi)\right|_{\xi=\xi_{0, j}}}+\frac{1}{2} \mathcal{C}_{\infty} \\
& =\frac{1}{2 \pi i} \mathcal{P} \int_{-\infty}^{+\infty} d \xi \frac{f(\xi)}{g(\xi)}+\frac{1}{2} \sum_{j=1}^{n+1} \int_{-\infty}^{+\infty} d \xi f(\xi) \frac{\delta\left(\xi-\xi_{0, j}\right)}{\left[g^{\prime}(\xi)\right]_{\xi=\xi_{0, j}}}+\frac{1}{2} \mathcal{C}_{\infty} \\
& =\frac{1}{2 \pi i} \mathcal{P} \int_{-\infty}^{+\infty} d \xi \frac{f(\xi)}{g(\xi)}+\frac{1}{2} f(\omega) \prod_{m=1}^{n} \frac{1}{\left(\omega-\omega_{m}\right)}+\frac{1}{2} \sum_{l=1}^{n} \frac{f\left(\omega_{l}\right)}{\left(\omega_{l}-\omega\right)} \prod_{m=1, m \neq l}^{n} \frac{1}{\left(\omega_{l}-\omega_{m}\right)}+\frac{1}{2} \mathcal{C}_{\infty},
\end{aligned}
$$

where $g(\xi)=(\xi-\omega) \prod_{m=1}^{n}\left(\xi-\omega_{m}\right), g^{\prime}(\xi)=\frac{d g(\xi)}{d \xi}, \mathcal{P}$ denotes the principal value of the integral along the real axis from $-\infty$ to $+\infty$, and $C$ denotes the contour drawn in Fig. 4. The contribution from the infinite semicircle is a complex quantity $\mathcal{C}_{\infty}=C_{\infty}+i C_{\infty}^{\prime}$. Note that we have also utilized the Dirac identity $\int d x \frac{f(x)}{x-x_{0}+i \eta}=\mathcal{P} \int d x \frac{f(x)}{x-x_{0}}-i \pi \int d x f(x) \delta\left(x-x_{0}\right)$ and $\operatorname{sgn}(x) /|x|=1 / x$.

From these two equations, we thus arrive at

$$
f(\omega) \prod_{m=1}^{n} \frac{1}{\left(\omega-\omega_{m}\right)}=\sum_{l=1}^{n} \frac{f\left(\omega_{l}\right)}{\left(\omega-\omega_{l}\right)} \prod_{m=1, m \neq l}^{n} \frac{1}{\left(\omega_{l}-\omega_{m}\right)}+\frac{1}{\pi i} \mathcal{P} \int_{-\infty}^{+\infty} d \xi \frac{f(\xi)}{g(\xi)}+\mathcal{C}_{\infty}
$$

whose real and imaginary parts are given as

$$
\begin{aligned}
& \operatorname{Re}[f(\omega)] \prod_{m=1}^{n} \frac{1}{\left(\omega-\omega_{m}\right)}=\sum_{l=1}^{n} \frac{\operatorname{Re} f\left(\omega_{l}\right)}{\left(\omega-\omega_{l}\right)} \prod_{m=1, m \neq l}^{n} \frac{1}{\left(\omega_{l}-\omega_{m}\right)}+\frac{1}{\pi} \mathcal{P} \int_{-\infty}^{+\infty} d \xi \frac{\operatorname{Im} f(\xi)}{g(\xi)}+C_{\infty}, \\
& \operatorname{Im}[f(\omega)] \prod_{m=1}^{n} \frac{1}{\left(\omega-\omega_{m}\right)}=\sum_{l=1}^{n} \frac{\operatorname{Im} f\left(\omega_{l}\right)}{\left(\omega-\omega_{l}\right)} \prod_{m=1, m \neq l}^{n} \frac{1}{\left(\omega_{l}-\omega_{m}\right)}-\frac{1}{\pi} \mathcal{P} \int_{-\infty}^{+\infty} d \xi \frac{\operatorname{Re} f(\xi)}{g(\xi)}+C_{\infty}^{\prime},
\end{aligned}
$$

which are named the Kramers-Kronig relation with $n$ th-order subtraction. It is one of the main results in this paper, if $f(\xi)$ does not diverge more than $\xi^{n-1}$ as $\xi \rightarrow \infty$, which leads to $\mathcal{C}_{\infty}=C_{\infty}+i C_{\infty}^{\prime}=0$.

Several remarks are in order here. First, for $n=0$ and $n=1$, our result recovers the conventional Kramers-Kronig relation and the one with first-order subtraction [62], respectively. Second, the quantities of $\omega_{1}, \omega_{2}, \omega_{3}, \ldots$, and $\omega_{n}$ can be arbitrarily chosen as if it is not equal to $q$. For the sake of simplicity, we choose $\omega_{j}=\alpha_{j} q$ with $\alpha_{i} \neq \alpha_{j} \neq 1$. Third, $\mathcal{J}_{m}$ can be used to calculate all the cases for $f(\xi) \approx \xi^{n}$ with $n \leqslant m, \ldots$. Finally, if a function $f(\xi)$ is $\sim \xi^{1}$, either $\mathcal{J}_{2}$ or $\mathcal{J}_{1}$ is applicable, but the latter is much convenient than the former, especially for the extrinsic parts.

\section{APPENDIX F: RELATIONS BETWEEN THE CORRELATION FUNCTIONS WITH OPPOSITE CHEMICAL POTENTIALS}

In this Appendix, we prove relations between chirality-dependent correlation functions with opposite chemical potentials. Next we take the antisymmetric part of $\Pi^{\alpha \beta}(\boldsymbol{q}, \omega, \chi)$ as an example to derive the relation of chirality-dependent correlation functions 
with opposite chemical potentials. For the positive chemical potential $+\mu_{\chi}$, we have

$$
F_{A}\left(\boldsymbol{q}, \omega, \chi, \mu_{\chi}\right)=\frac{1}{2 \mathcal{V}} \sum_{\boldsymbol{k}} \sum_{\lambda, \lambda^{\prime}= \pm}\left(\lambda \frac{(\boldsymbol{k}+\boldsymbol{q})^{2}-\boldsymbol{k}^{2}-q^{2}}{2 q^{2}|\boldsymbol{k}|}-\lambda^{\prime} \frac{(\boldsymbol{k}+\boldsymbol{q})^{2}-\boldsymbol{k}^{2}+q^{2}}{2 q^{2}|\boldsymbol{k}+\boldsymbol{q}|}\right) \frac{n_{F}\left[\varepsilon_{\lambda}(\boldsymbol{k})\right]-n_{F}\left[\varepsilon_{\lambda^{\prime}}(\boldsymbol{k}+\boldsymbol{q})\right]}{\omega+\varepsilon_{\lambda}(\boldsymbol{k})-\varepsilon_{\lambda^{\prime}}(\boldsymbol{k}+\boldsymbol{q})+i \eta},
$$

while for the negative chemical potential $-\mu_{\chi}$,

$$
\begin{aligned}
F_{A}\left(\boldsymbol{q}, \omega, \chi,-\mu_{\chi}\right) & =\frac{1}{2 \mathcal{V}} \sum_{\boldsymbol{k}} \sum_{\lambda, \lambda^{\prime}= \pm}\left(\lambda \frac{(\boldsymbol{k}+\boldsymbol{q})^{2}-\boldsymbol{k}^{2}-q^{2}}{2 q^{2}|\boldsymbol{k}|}-\lambda^{\prime} \frac{(\boldsymbol{k}+\boldsymbol{q})^{2}-\boldsymbol{k}^{2}+q^{2}}{2 q^{2}|\boldsymbol{k}+\boldsymbol{q}|}\right) \frac{\tilde{n}_{F}\left[\varepsilon_{\lambda}(\boldsymbol{k})\right]-\tilde{n}_{F}\left[\varepsilon_{\lambda^{\prime}}(\boldsymbol{k}+\boldsymbol{q})\right]}{\omega+\varepsilon_{\lambda}(\boldsymbol{k})-\varepsilon_{\lambda^{\prime}}(\boldsymbol{k}+\boldsymbol{q})+i \eta} \\
& =\frac{1}{2 \mathcal{V}} \sum_{\boldsymbol{k}} \sum_{\lambda, \lambda^{\prime}= \pm}\left(\lambda \frac{(\boldsymbol{k}+\boldsymbol{q})^{2}-\boldsymbol{k}^{2}-q^{2}}{2 q^{2}|\boldsymbol{k}|}-\lambda^{\prime} \frac{(\boldsymbol{k}+\boldsymbol{q})^{2}-\boldsymbol{k}^{2}+q^{2}}{2 q^{2}|\boldsymbol{k}+\boldsymbol{q}|}\right) \frac{n_{F}\left[-\varepsilon_{\lambda^{\prime}}(\boldsymbol{k}+\boldsymbol{q})\right]-n_{F}\left[-\varepsilon_{\lambda}(\boldsymbol{k})\right]}{\omega-\varepsilon_{\lambda^{\prime}}(\boldsymbol{k}+\boldsymbol{q})+\varepsilon_{\lambda}(\boldsymbol{k})+i \eta},
\end{aligned}
$$

where $\tilde{n}_{F}[x]=1 /\left[\exp \left\{\beta_{T}\left(x+\mu_{\chi}\right)\right\}+1\right]$ and $\tilde{n}_{F}[x]+n_{F}[-x]=1$. Because of the particle-hole symmetry for each Weyl node, the energy dispersion relation satisfies $\varepsilon_{\lambda}(\boldsymbol{k})=-\varepsilon_{-\lambda}(\boldsymbol{k})$ such that

$$
F_{A}\left(\boldsymbol{q}, \omega, \chi,-\mu_{\chi}\right)=\frac{1}{2 \mathcal{V}} \sum_{\boldsymbol{k}} \sum_{\lambda, \lambda^{\prime}= \pm}\left(\lambda \frac{(\boldsymbol{k}+\boldsymbol{q})^{2}-\boldsymbol{k}^{2}-q^{2}}{2 q^{2}|\boldsymbol{k}|}-\lambda^{\prime} \frac{(\boldsymbol{k}+\boldsymbol{q})^{2}-\boldsymbol{k}^{2}+q^{2}}{2 q^{2}|\boldsymbol{k}+\boldsymbol{q}|}\right) \frac{n_{F}\left[\varepsilon_{-\lambda^{\prime}}(\boldsymbol{k}+\boldsymbol{q})\right]-n_{F}\left[\varepsilon_{-\lambda}(\boldsymbol{k})\right]}{\omega+\varepsilon_{-\lambda^{\prime}}(\boldsymbol{k}+\boldsymbol{q})-\varepsilon_{-\lambda}(\boldsymbol{k})+i \eta} .
$$

Relabeling $(\boldsymbol{k}, \lambda) \leftrightarrow\left(-\boldsymbol{k}-\boldsymbol{q},-\lambda^{\prime}\right)$ leads to

$$
F_{A}\left(\boldsymbol{q}, \omega, \chi,-\mu_{\chi}\right)=\frac{1}{2 \mathcal{V}} \sum_{\boldsymbol{k}} \sum_{\lambda, \lambda^{\prime}= \pm}\left(-\lambda^{\prime} \frac{\boldsymbol{k}^{2}-(\boldsymbol{k}+\boldsymbol{q})^{2}-q^{2}}{2 q^{2}|\boldsymbol{k}+\boldsymbol{q}|}+\lambda \frac{\boldsymbol{k}^{2}-(\boldsymbol{k}+\boldsymbol{q})^{2}+q^{2}}{2 q^{2}|\boldsymbol{k}|}\right) \frac{n_{F}\left[\varepsilon_{\lambda}(\boldsymbol{k})\right]-n_{F}\left[\varepsilon_{\lambda^{\prime}}(\boldsymbol{k}+\boldsymbol{q})\right]}{\omega+\varepsilon_{\lambda}(\boldsymbol{k})-\varepsilon_{\lambda^{\prime}}(\boldsymbol{k}+\boldsymbol{q})+i \eta} .
$$

Clearly, $F_{A}\left(\boldsymbol{q}, \omega, \chi,-\mu_{\chi}\right)$ is nothing but $-F_{A}\left(\boldsymbol{q}, \omega, \chi,+\mu_{\chi}\right)$. Other chirality-dependent correlation functions can be calculated in a parallel way. Finally, we have

$$
\begin{gathered}
\Pi^{00}\left(\boldsymbol{q}, \omega, \chi,-\mu_{\chi}\right)=\Pi^{00}\left(\boldsymbol{q}, \omega, \chi, \mu_{\chi}\right), \\
F_{X}\left(\boldsymbol{q}, \omega, \chi,-\mu_{\chi}\right)=F_{X}\left(\boldsymbol{q}, \omega, \chi, \mu_{\chi}\right), \\
F_{A}\left(\boldsymbol{q}, \omega, \chi,-\mu_{\chi}\right)=-F_{A}\left(\boldsymbol{q}, \omega, \chi, \mu_{\chi}\right),
\end{gathered}
$$

where $X=T, L, I$. Note that the relation of $\Pi^{00}$ is consistent with our previous result [47].

[1] P. Hosur and X. Qi, C. R. Phys. 14, 857 (2013).

[2] A. Burkov, J. Phys.: Condens. Matter 27, 113201 (2015).

[3] H. Weng, X. Dai, and Z. Fang, J. Phys.: Condens. Matter 28, 303001 (2016).

[4] N. P. Armitage, E. J. Mele, and A. Vishwanath, Rev. Mod. Phys. 90, 015001 (2018).

[5] D. Xiao, M.-C. Chang, and Q. Niu, Rev. Mod. Phys. 82, 1959 (2010).

[6] G. E. Volovik, The Universe in a Helium Droplet (Oxford University Press, Oxford, UK, 2003).

[7] H. Nielsen and M. Ninomiya, Phys. Lett. B 130, 389 (1983).

[8] P. Goswami and S. Tewari, Phys. Rev. B 88, 245107 (2013).

[9] C.-X. Liu, P. Ye, and X.-L. Qi, Phys. Rev. B 87, 235306 (2013).

[10] S. A. Parameswaran, T. Grover, D. A. Abanin, D. A. Pesin, and A. Vishwanath, Phys. Rev. X 4, 031035 (2014).

[11] J. Behrends, A. G. Grushin, T. Ojanen, and J. H. Bardarson, Phys. Rev. B 93, 075114 (2016).

[12] A. Vilenkin, Phys. Rev. D 22, 3080 (1980).

[13] K. Fukushima, D. E. Kharzeev, and H. J. Warringa, Phys. Rev. D 78, 074033 (2008).

[14] A. G. Grushin, Phys. Rev. D 86, 045001 (2012).

[15] A. A. Zyuzin and A. A. Burkov, Phys. Rev. B 86, 115133 (2012).
[16] J. Zhou, H. Jiang, Q. Niu, and J. Shi, Chin. Phys. Lett. 30, 027101 (2013).

[17] M. M. Vazifeh and M. Franz, Phys. Rev. Lett. 111, 027201 (2013).

[18] K. Landsteiner, Phys. Rev. B 89, 075124 (2014).

[19] M.-C. Chang and M.-F. Yang, Phys. Rev. B 91, 115203 (2015).

[20] T. E. O'Brien, C. W. J. Beenakker, and I. Adagideli, Phys. Rev. Lett. 118, 207701 (2017).

[21] D. E. Kharzeev and H. J. Warringa, Phys. Rev. D 80, 034028 (2009).

[22] D. T. Son and N. Yamamoto, Phys. Rev. D 87, 085016 (2013).

[23] J. Ma and D. A. Pesin, Phys. Rev. B 92, 235205 (2015).

[24] S. Zhong, J. E. Moore, and I. Souza, Phys. Rev. Lett. 116, 077201 (2016).

[25] X. Wan, A. M. Turner, A. Vishwanath, and S. Y. Savrasov, Phys. Rev. B 83, 205101 (2011).

[26] D. T. Son and B. Z. Spivak, Phys. Rev. B 88, 104412 (2013).

[27] A. A. Burkov, Phys. Rev. Lett. 113, 247203 (2014).

[28] E. V. Gorbar, V. A. Miransky, and I. A. Shovkovy, Phys. Rev. B 89, 085126 (2014).

[29] Y. Gao, S. A. Yang, and Q. Niu, Phys. Rev. B 95, 165135 (2017).

[30] X. Dai, Z. Z. Du, and H.-Z. Lu, Phys. Rev. Lett. 119, 166601 (2017). 
[31] H.-J. Kim, K.-S. Kim, J.-F. Wang, M. Sasaki, N. Satoh, A. Ohnishi, M. Kitaura, M. Yang, and L. Li, Phys. Rev. Lett. 111, 246603 (2013).

[32] X. Huang, L. Zhao, Y. Long, P. Wang, D. Chen, Z. Yang, H. Liang, M. Xue, H. Weng, Z. Fang, X. Dai, and G. Chen, Phys. Rev. X 5, 031023 (2015).

[33] J. Xiong, S. K. Kushwaha, T. Liang, J. W. Krizan, M. Hirschberger, W. Wang, R. J. Cava, and N. P. Ong, Science 350, 413 (2015).

[34] C.-Z. Li, L.-X. Wang, H. Liu, J. Wang, Z.-M. Liao, and D.-P. Yu, Nat. Commun. 6, 10137 (2015).

[35] H. Li, H. He, H.-Z. Lu, H. Zhang, H. Liu, R. Ma, Z. Fan, S.-Q. Shen, and J. Wang, Nat. Commun. 7, 10301 (2016).

[36] Q. Li, D. E. Kharzeev, C. Zhang, Y. Huang, I. Pletikosic, A. V. Fedorov, R. D. Zhong, J. A. Schneeloch, G. D. Gu, and T. Valla, Nat. Phys. 12, 550 (2016).

[37] D. Foster, Hydrodynamic Fluctuations, Broken Symmetry, and Correlation Functions (Benjamin-Cummings, Reading, 1975).

[38] G. Giuliani and G. Vignale, Quantum Theory of the Electron Liquid (Cambridge University Press, Cambridge, UK, 2005).

[39] G. D. Mahan, Many-Particle Physics, 3rd ed. (Springer, New York, 2007).

[40] N. Nagaosa, J. Sinova, S. Onoda, A. H. MacDonald, and N. P. Ong, Rev. Mod. Phys. 82, 1539 (2010).

[41] L. D. Landau and E. M. Lifshitz, Electrodynamics of Continuous Media, 2nd ed. (Pergamon Press, New York, 1984).

[42] G. Passarino and M. Veltman, Nucl. Phys. B 160, 151 (1979).

[43] H.-R. Chang, J. Zhou, S.-X. Wang, W.-Y. Shan, and D. Xiao, Phys. Rev. B 92, 241103 (2015).

[44] In this paper, we mainly consider the case in which $\boldsymbol{b}=0$ or $|\boldsymbol{b}|$ is sufficiently great so that Weyl fermions near the two Weyl nodes with opposite chirality behave independently in the presence of external fields.

[45] A. A. Abrikosov and S. D. Beneslavskii, Sov. Phys. JETP 32, 699 (1971).

[46] M. Lv and S.-C. Zhang, Int. J. Mod. Phys. B 27, 1350177 (2013).

[47] J. Zhou, H.-R. Chang, and D. Xiao, Phys. Rev. B 91, 035114 (2015).

[48] J. Hofmann and S. Das Sarma, Phys. Rev. B 91, 241108 (2015).

[49] D. E. Kharzeev, R. D. Pisarski, and H.-U. Yee, Phys. Rev. Lett. 115, 236402 (2015).

[50] F. M. D. Pellegrino, M. I. Katsnelson, and M. Polini, Phys. Rev. B 92, 201407 (2015).

[51] A. A. Zyuzin and V. A. Zyuzin, Phys. Rev. B 92, 115310 (2015).

[52] O. V. Kotov and Y. E. Lozovik, Phys. Rev. B 93, 235417 (2016).

[53] Y. Ferreiros and A. Cortijo, Phys. Rev. B 93, 195154 (2016).

[54] Z. Yan, P.-W. Huang, and Z. Wang, Phys. Rev. B 93, 085138 (2016).
[55] Z. Song, J. Zhao, Z. Fang, and X. Dai, Phys. Rev. B 94, 214306 (2016).

[56] P. Rinkel, P. L. S. Lopes, and I. Garate, Phys. Rev. Lett. 119, 107401 (2017).

[57] D. Liu and J. Shi, Phys. Rev. Lett. 119, 075301 (2017).

[58] Y. Araki and K. Nomura, Phys. Rev. B 93, 094438 (2016).

[59] The general finite-temperature correlation functions of $3 \mathrm{D}$ Weyl/Dirac semimetals cannot be entirely expressed in terms of elementary functions. However, an elegant approach might be useful to connect the finite-temperature correlation functions to the zero-temperature ones (see Sec. 4.4.4 in Ref. [38]).

[60] M. Peskin and D. Schroeder, An Introduction to Quantum Field Theory (Addison-Wesley, New York, 1995).

[61] V. N. Kotov, B. Uchoa, V. M. Pereira, F. Guinea, and A. H. Castro Neto, Rev. Mod. Phys. 84, 1067 (2012).

[62] J. Bjorken and S. Drell, Relativistic Quantum Fields (McGrawHill, New York, 1965).

[63] T. Stauber, D. Noriega-Pérez, and J. Schliemann, Phys. Rev. B 91, 115407 (2015).

[64] C. J. Tabert, J. P. Carbotte, and E. J. Nicol, Phys. Rev. B 93 085426 (2016).

[65] B. Roy, V. Juricic, and S. Das Sarma, Sci. Rep. 6, 32446 (2016).

[66] B. Roy, P. Goswami, and V. Juričić, Phys. Rev. B 95, 201102 (2017).

[67] W. A. Bardeen, Phys. Rev. 184, 1848 (1969).

[68] W. A. Bardeen and B. Zumino, Nucl. Phys. B 244, 421 (1984).

[69] E. V. Gorbar, V. A. Miransky, I. A. Shovkovy, and P. O. Sukhachov, Phys. Rev. Lett. 118, 127601 (2017).

[70] Z.-M. Huang, J. Zhou, and S.-Q. Shen, Phys. Rev. B 96, 085201 (2017).

[71] M. Koshino and I. F. Hizbullah, Phys. Rev. B 93, 045201 (2016).

[72] P. J. W. Moll, A. C. Potter, N. L. Nair, B. J. Ramshaw, K. A. Modic, S. Riggs, B. Zeng, N. J. Ghimire, E. D. Bauer, R. Kealhofer, F. Ronning, and J. G. Analytis, Nat. Commun. 7, 12492 (2016).

[73] Within the linear model for Weyl fermions, the anomalous Hall conductivity keeps unaltered at a finite density, which is consistent with the fact that the chiral anomaly is unaffected by a finite chemical potential [74].

[74] L. Dolan and R. Jackiw, Phys. Rev. D 9, 3320 (1974).

[75] A. Principi, M. Polini, and G. Vignale, Phys. Rev. B 80, 075418 (2009).

[76] A. Scholz and J. Schliemann, Phys. Rev. B 83, 235409 (2011).

[77] R. E. Raab and O. L. De Lange, Multipole Theory in Electromagnetism: Classical, Quantum, and Symmetry Aspects, with Applications (Oxford University Press, Oxford, UK, 2005).

[78] A. Thakur, K. Sadhukhan, and A. Agarwal, Phys. Rev. B 97, 035403 (2018). 